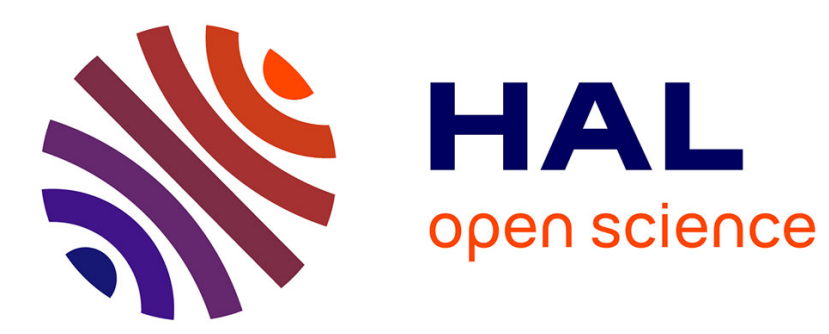

\title{
Mixed-Mode Oscillations in a piecewise linear system with multiple time scale coupling
}

Soledad Fernández-García, Maciej Krupa, Frédérique Clément

\section{To cite this version:}

Soledad Fernández-García, Maciej Krupa, Frédérique Clément. Mixed-Mode Oscillations in a piecewise linear system with multiple time scale coupling. Physica D: Nonlinear Phenomena, 2016, 332, pp.9-22. 10.1016/j.physd.2016.06.002 . hal-01342978

\section{HAL Id: hal-01342978 \\ https://inria.hal.science/hal-01342978}

Submitted on 7 Jul 2016

HAL is a multi-disciplinary open access archive for the deposit and dissemination of scientific research documents, whether they are published or not. The documents may come from teaching and research institutions in France or abroad, or from public or private research centers.
L'archive ouverte pluridisciplinaire HAL, est destinée au dépôt et à la diffusion de documents scientifiques de niveau recherche, publiés ou non, émanant des établissements d'enseignement et de recherche français ou étrangers, des laboratoires publics ou privés. 


\title{
Mixed-Mode Oscillations in a piecewise linear system with multiple time scale coupling
}

\author{
S. Fernández-García ${ }^{\mathrm{a}, *}$, M. Krupa ${ }^{\mathrm{b}}$, F. Clément $^{\mathrm{a}}$ \\ ${ }^{a}$ Inria Paris Research Centre, 2 rue Simone Iff, CS 42112 75589, Paris Cedex 12 \\ ${ }^{b}$ Inria Sophia Antipolis Méditerranée Research Center, 2004 Route des Lucioles, 06902 \\ Valbonne, France
}

\begin{abstract}
In this work, we analyze a four dimensional slow-fast piecewise linear system with three time scales presenting Mixed-Mode Oscillations. The system possesses an attractive limit cycle along which oscillations of three different amplitudes and frequencies can appear, namely, small oscillations, pulses (medium amplitude) and one surge (largest amplitude). In addition to proving the existence and attractiveness of the limit cycle, we focus our attention on the canard phenomena underlying the changes in the number of small oscillations and pulses. We analyze locally the existence of secondary canards leading to the addition or subtraction of one small oscillation and describe how this change is globally compensated for or not with the addition or subtraction of one pulse.
\end{abstract}

Edited final version published in: Physica D, 2016, 332: 9-22

http://dx.doi.org/10.1016/j.physd.2016.06.002

Keywords: piecewise linear systems, slow-fast dynamics, coupled oscillators, secondary canards, mixed-mode oscillations 2000 MSC: 34C15, 34C25, 34C26, 70K70, 93C70

\footnotetext{
${ }^{*}$ Corresponding author. Present address: Dpto, E.D.A.N., Universidad de Sevilla, Aptdo. 1160, 41080 Sevilla, Spain. The first author is supported by University of Seville VPPI-US under grant PPI2015-II.5, and partially supported by Proyectos de Excelencia de la Junta de Andalucía under grant P12-FQM-1658 and Ministerio de Economía y Competitividad under grant MTM2015-65608-P.

Email addresses: soledad@us.es (S. Fernández-García), maciej.krupa@inria.fr (M. Krupa), frederique.clement@inria.fr (F. Clément)
} 


\section{Introduction}

In [13], we considered a piecewise linear (PWL) version of the system initially proposed in $[1,2]$, a model introduced in a context of mathematical neuroendocrinology. The original smooth model in $[1,2]$ consists of a four dimensional (4D) system made of two coupled FitzHugh-Nagumo subsystems running on different timescales, in which the fast subsystem is forced by the slow subsystem. We replaced in [13] the original FitzHugh-Nagumo subsystems $[5,7]$ by two PWL equivalents (McKean caricatures [6]). This change allowed us to obtain more information on the dynamics as well as a more direct control of the quantitative features of the system output. A typical orbit of the PWL model possesses a periodic behavior with oscillations of two different amplitudes and frequencies: a series of pulses (medium amplitude) and one surge (larger amplitude).

The smooth model, with three different time scales, can display MixedMode Oscilations (MMOs) of three different types [3]. Apart from the pulses and surge, a pause may exist between the end of the surge and the resumption of the pulses, where small oscillations take place, see Fig. 1 in [3]. However, the McKean caricatures do not capture the existence of such small oscillations. Here, we propose an extension of the model considered in [13]. In the new extended model, small oscillations can happen, see Fig. 1. Such small oscillations are underlain by the existence of a canard explosion in the fast system. To obtain a proper canard explosion in a PWL system, one small extra linearity zone is necessary, as it has been pointed out in $[12,18,9]$.

The present work focuses on the mechanisms underlying the creationdestruction of the resulting small oscillations and describes how the addition or loss of one small oscillation after the surge is possibly compensated for by the loss or addition of one pulse before the surge. In the smooth model, such a compensation mechanism has been explored numerically [3] and it turned out to be too complicated to be studied analytically. The PWL nature of the system proposed in this work, allows us to better understand the dynamical mechanisms than in the case of the smooth model. More specifically, we find two remarkable results. First, the number of small oscillations needed to be lost in order to win one pulse increases while we decrease the intermediate time scale of the system. Second, as the system goes through the canard explosion, one pulse disappears at the end of the pulsatility phase and another is created from a small oscillation at the beginning of the pulsatility phase. We describe in detail these facts along the paper and more specifically in 
Proposition 3 and Theorem 3. Moreover, fixing the time scales, we are able to control the number of small oscillations as well as the addition or subtraction of pulses just by tuning a regular parameter of the system.

We note that our method of counting small oscillations relies heavily on the presence of an attracting limit cycle which includes the sequence of small oscillations as a part of its trajectory. A more general method for counting small oscillations and computing secondary canards is provided in [9]. The main novelty of our paper is the study of the interaction of two canard phenomena, both occurring dynamically, one at the corner point of the fast nullcline and another at the small zone replacing the other critical point, see Figure 2. The context of PWL systems, due to its simplicity and the availability of explicit formulas, provides a suitable context for this study.

Here, we consider the following class of 4D PWL slow-fast systems,

$$
\begin{aligned}
& \left\{\begin{array}{l}
\dot{x}=-y+f(x), \\
\dot{y}=\delta\left(x+a_{2}+c X\right),
\end{array}\right. \\
& \left\{\begin{array}{l}
\dot{X}=\delta(-Y+g(X)), \\
\dot{Y}=\varepsilon \delta\left(X+b_{1} Y+b_{2}\right),
\end{array}\right.
\end{aligned}
$$

where

$$
f(x)= \begin{cases}-x-2, & \text { if } x \leq-1 \\ x, & \text { if } x \in(-1,1-\sqrt{\delta}] \\ 1-\sqrt{\delta}, & \text { if } x \in(1-\sqrt{\delta}, 1+\sqrt{\delta}) \\ -x+2, & \text { if } x \geq 1+\sqrt{\delta}\end{cases}
$$

and

$$
g(X)= \begin{cases}-X-k_{2}-1, & \text { if } X \leq-1 \\ k_{2} X, & \text { if }|X| \leq 1 \\ -X+k_{2}+1, & \text { if } X \geq 1\end{cases}
$$

with $(x, y, X, Y)^{T} \in \mathbb{R}^{4}, 0<\varepsilon, \delta \ll 1, b_{1} \geq 0, a_{2}, b_{2}, c, k_{1}, k_{2}$ are real and positive, and the overdot denotes the derivative with respect to time variable $t$. Let us call the zones of subsystem (1) the left, center, extra or right zone, when the corresponding first variable $x$ is, respectively, smaller that -1 , between -1 and $1-\sqrt{\delta}$, between $1-\sqrt{\delta}$ and $1+\sqrt{\delta}$ or larger than $1+\sqrt{\delta}$ (see the PWL-nullcline in Fig. 2). Sometimes we will use the superscripts $L, C, E$ and $R$, to specify the referred zone. Note that the size of the extra zone $(2 \sqrt{\delta})$ has been chosen following [12] in order to give rise to a canard explosion in subsystem (1). In the same way, we call the zones of subsystem 
(2) the left, center, or right zone, when the corresponding first variable $X$ is smaller than -1 , between -1 and 1 or larger than 1 . We also use the superscripts $L, C$ and $R$, to specify the referred zone. Note that, for the sake of simplicity, we study here the case where the $y$-nullcline is vertical.

System (1)-(2) consists of two coupled planar oscillators, one in variables $x, y$ and the other in variables $X, Y$. The coupling is one-way, that is, the subsystem in variables $X, Y$ evolves independently and we call it the forcing system. Variable $X$ is forcing the $(x, y) 2 \mathrm{D}$ system by changing dynamically the location of the $y$-nullcline.

System (1)-(2) is slow-fast with three time scales, namely, $1, \delta$ and $\varepsilon \delta$. The forcing system $(X, Y)$ evolves more slowly than the forced system $(x, y)$. Bearing this in mind, we call the forcing system the slow system and the forced system the fast system. Moreover, each of the two planar systems is also slow-fast, with slow variable $y$ and $Y$, respectively, and fast variable $x$ and $X$, respectively.

Let us first consider some assumptions, in order to obtain the MMOs which were observed in the smooth system in [3], and explain briefly the dynamical behavior of the system.

(H1) The forcing system (2) has a relaxation limit cycle. We can divide the limit cycle into four parts, (see Fig. 3):

I. $X \in\left(X_{\min },-1\right), Y<g(X)$,

II. $X \in\left(-1, X_{\max }\right), Y<g(X)$,

III. $X \in\left(1, X_{\max }\right), Y>g(X)$,

IV. $X \in\left(X_{\min }, 1\right), Y>g(X)$.

Note that, as $\varepsilon \rightarrow 0$ the cycle is approaching the $X$-nullcline in the right and left zones and jumps almost horizontally when it reaches the middle zone. We use the approximation

$X_{\min } \simeq-2 k_{2}-1-\varepsilon\left(1+b_{2}+b_{1} k_{2}\right) \quad$ and $\quad X_{\max } \simeq 2 k_{2}+1+\varepsilon\left(1-b_{2}+b_{1} k_{2}\right)$,

valid for $\varepsilon$ small enough.

(H2) $\left|a_{2}-1\right|<c$.

Under hypothesis (H2), the vertical nullcline crosses the separation line $x=-1$ rightwards at a time when $|X|<1$, therefore, when $X$ 
(considered as the bifurcation parameter) travels along the limit cycle with the fast motion, see figures 3 and 9 . At the crossing time, a left canard superexplosion bifurcation occurs, that is, the system presents a bounded continuum of canard homoclinic orbits from the equilibrium point and by moving $X$ in such a way that the equilibrium enters the central zone, one large relaxation oscillation limit cycle is created (see figures 4 and 5 in [4]). The term superexplosion comes from the fact that instead of successive canard cycles curves ending up in the relaxation cycle, in this case, there is a continuum of canard homoclinic cycles that take place all at the same time, and the relaxation cycle is born instantaneously from this continuum. For more details about the canard superexplosion bifurcation see [4].

Here, in contrast to [13], we do not impose any upper bound for $c$. As a consequence, the rightmost location of the $y$-nullcline can reach the right zone, hence a right canard explosion bifurcation (actually, the PWL equivalent) [12] can occur while $X$ is along the relaxation limit cycle, see Figure 9. The right canard explosion bifurcation takes place when the equilibrium point is located in the extra zone, that is, $x \in(1-\sqrt{\delta}, 1+\sqrt{\delta})$. We explain this case in detail in the following lines.

Remark 1. Note that, as it was explained in detail in Remark 4 of [13], tuning parameter $b_{1}$ enables us to adjust the pulse frequency, more specifically, the duration of the low-frequency part of the pulsatile regime with respect to that of the high frequency part. As in this article we are no more interested in fitting our model to biological specifications, we have fixed $b_{1}=0$ along all the simulations. Regarding parameter $a_{2}$, we only need to be sure that hypothesis (H2) $\left|a_{2}-1\right|<c$ is satisfied. We have chosen $a_{2}=2$ because in this case (H2) reads $c>1$, but another value could be equally chosen.

The value of $X$ determines the location of the $y$-nullcline, hence the location of the unique equilibrium point of the fast system (see Fig. 8, for instance). If the value of $X$ is such that the equilibrium point is located in zone $R$ or $L$, then the fast system has a stable node and the orbits are approaching it. If the location of $X$ is such that the equilibrium point is located in the central zone, then the orbits oscillate across the four linearity zones around the equilibrium point. Finally, if the location of $X$ is such that 
the equilibrium point is located in the extra zone, the system has a zonal linear center completely contained in the extra zone.

Note that, in [13], we imposed a superior bound for $c$, to prevent the right canard superexplosion from occurring. In that case, the system alternates between a quasi-stationary regime, called surge, and an oscillatory regime, called pulsatility phase, see Fig. 4. Here, we are interested in reproducing the small oscillations occurring after the surge (see Fig. 1). To this aim, we need to let the $y$-nullcline pass through the upper corner, to guarantee the occurrence of the canard phenomena. Let us remark again that, if the $x$-nullcline does not have the extra piece, the system is not able to reproduce small oscillations, even if the $y$-nullcline passes through the corner [4]. As the system without the extra piece has already been analyzed in [13], we focus our attention on the new dynamics guaranteed by the extra piece.

The coefficient matrix in the extra zone is

$$
A^{E}=\left(\begin{array}{cc}
0 & -1 \\
\delta & 0
\end{array}\right)
$$

and the linear configuration type is a center. Note that one can also consider a non-zero trace configuration, giving rise to a stable or unstable focus. In order to have a canard explosion, we would need this trace to be of the order of $O(\sqrt{\delta})$, see [12]. The analysis considered in this paper can be adapted to that case. The only difference would concern the shape of the small oscillations, whose amplitude would either increase or decrease according to the stability of the focus. To understand the dynamics of the whole system with the extra piece, it is easier to consider first independently the dynamical behavior of system (1) for static positions of its vertical nullcline, while $X$ is in phase I of the relaxation cycle.

Let us consider a new parameter $a \in \mathbb{R}$ and denote

$$
X_{a}=\frac{-a_{2}-a}{c}
$$

the value of $X$ such that the $y$-nullcline is $x=a$. Note that, in any case, the forced system has only one equilibrium point whose first coordinate is given by $x=a$. According to the value of $a$, there are different dynamical configurations, as we explain subsequently. For the sake of clarity, we illustrate in figures 5 to 7 the three dynamically different phase planes that we can find when the $y$-nullcline is located in the extra zone, that is, $a \in(1-\sqrt{\delta}, 1+\sqrt{\delta})$. 
1. $a<-1$. The unique equilibrium point of the system is located in the left zone and it is a stable node.

2. $a=-1$. The unique equilibrium point of the system is located on the boundary between the central and left zones. On the central side it is a unstable node and on the left side it is a stable node. As it has been explained before, the system presents a bounded continuum of canard homoclinic orbits.

3. $a \in(-1,1-\sqrt{\delta})$. The fast system possesses a relaxation limit cycle surrounding the only equilibrium point of the system, which is located in the central zone, and it is a unstable node.

4. $a=1-\sqrt{\delta}$. The unique equilibrium point of the system is located on the boundary between the central and extra zones. On the central side it is a unstable node and on the extra side it is a center. There exists a relaxation limit cycle surrounding the unique equilibrium point.

5. $a \in(1-\sqrt{\delta}, 1)$. The system has a repulsive zonal linear center bounded by the periodic orbit tangent to the boundary $x=1-\sqrt{\delta}$, completely contained in the extra zone. There exists a relaxation limit cycle surrounding the zonal linear center, see Fig. 5.

6. $a=1$. The system has an attractive continuum of periodic orbits surrounding the equilibrium, which in this case is located at $(x, y)=$ $(1,1-\sqrt{\delta})$. The continuum is bounded by the maximal canard [12], see Fig. 6 .

7. $a \in(1,1+\sqrt{\delta})$. The system has an attractive zonal linear center completely contained in the extra zone, and bounded by the periodic orbit tangent to the boundary $x=1+\sqrt{\delta}$, see Fig. 7 .

8. $a=1+\sqrt{\delta}$. The unique equilibrium point of the system is located on the boundary between the right and extra zones. On the right side it is a stable node and on the extra side it is a center. It is a global attractor of the system.

9. $a>1+\sqrt{\delta}$. The unique equilibrium point of the system is located in the right zone and it is a stable node. 
Remark 2. The existence of a stable relaxation cycle in previous statements 3, 4 and 5 is obtained from the application of the slow-fast theory, similarly as in the case of smooth slow-fast systems, see for example [15]. Given both the slow and fast dynamics, and the dynamics in the extra zone, any recurrent dynamics exiting the extra zone must follow the two stable slow manifolds and therefore must be equal to the relaxation cycle itself. Such a result is nevertheless only valid for $\delta$ sufficiently small.

Having in mind the phase portraits of fast system (1) for static positions of the vertical nullcline, as just described, we now consider the dynamics of the whole 4D system (1)-(2). Depending on the rightmost position of the vertical nullcline while $X$ is along the relaxation cycle, the $4 \mathrm{D}$ system presents different dynamics, yet in any case, it possesses an attractive limit cycle, as we establish in the following result, whose proof has been relegated to Section 4.

Theorem 1. Consider system (1)-(2) with $\varepsilon$ and $\delta$ small enough, under hypotheses (H1) and (H2). There exists a unique attractive limit cycle $\gamma_{\boldsymbol{\mu}}$, where $\boldsymbol{\mu}=\left(\varepsilon, \delta, b_{1}, b_{2}, a_{2}, k_{2}, c\right)$, which can be of different types, depending on the rightmost position of the vertical nullcline of fast system (1), given by $-a_{2}-c X_{\min }$, with $X_{\min }$ approximated in (4):

1. If the rightmost position of the vertical nullcline is located in the center zone, the limit cycle consists of a series of pulses and one surge.

2. If the rightmost position of the vertical nullcline is located in the extra zone or in the right zone but close enough to the extra zone, a pause takes place between the surge and pulsatile regime. On the pause, small oscillations can occur and the limit cycle consists of a series of small oscillations, a series of pulses and one surge.

The period of limit cycle $\gamma_{\boldsymbol{\mu}}$ corresponds to the period of the relaxation cycle of the forcing system (2), computed in [13]. This period divides into two parts: the time when $X<0$ on the relaxation cycle of the forcing system, which corresponds to the small oscillations and pulse regime, and the time when $X>0$ on the relaxation cycle of the forcing system, which corresponds to the surge regime.

Note that the small oscillations appear in the vicinity of the extra piece of $y=f(x)$ at the beginning of phase I of the relaxation cycle of the forcing 
system (2), see Fig. 3 again. In case 2, some exceptional limit cycles, that we compute in Theorem 2, contain canard segments. In that result, we prove how varying a regular parameter can lead to a change in the number of small oscillations by means of a passage through the (upper) canard explosion. On the other hand, the passage through the lower canard superexplosion is responsible for the adding or subtraction of one pulse.

The rest of the article is outlined as follows. In Section 2, we focus on the computation of the number of small oscillations of the limit cycle during the pause, depending on the rightmost position of the vertical nullcline and their passage through the (upper) canard explosion. Section 3 is devoted to the global adding-subtraction of small oscillations and pulses. Section 4 is dedicated to the proof of Theorem 1. Finally, in Section 5 we present some conclusions and possible extensions of the present work.

\section{Approximation of the number of small oscillations during the pause}

\subsection{Extreme positions of the vertical nullcline of the fast system}

The number of small oscillations before the pulsatile regime depends mainly on the rightmost position of the $y$-nullcline while $X$ is along the relaxation cycle of the forcing system (2). When counting small oscillations and pulses, we consider that the limit cycle possesses one small oscillation (or pulse) when $\dot{y}$ vanishes passing from negative to positive slope. For instance, in the case represented in Fig. 1, we consider that the orbit possesses 5 small oscillations and 11 pulses. Note that the beginning of the surge is not taken into account when counting the pulses.

To begin with, we observe that if the rightmost position of the $y$-nullcline is such that the maximal canard of fast system (1) has not taken place $(a=1$, see Fig. 8), then there are no small oscillations before the pulses.

Proposition 1. Consider system (1)-(2) with $\varepsilon$ and $\delta$ small enough, under hypotheses (H1) and (H2). If

$$
c<\frac{a_{2}+1}{2 k_{2}+1}
$$

then the unique attractive limit cycle $\gamma_{\boldsymbol{\mu}}$ does not present any small oscillation before the pulsatile regime. 
Proof. For $X=X_{1}:=\left(-a_{2}-1\right) / c$, the $y$-nullcline of fast system (1) is $x=1$, that is, it is located exactly where a continuum of periodic orbits bounded by the maximal canard occurs, see Fig.6. If

$$
X_{\min }>X_{1}
$$

where $X_{\min }$ is the minimal value reached by variable $X$ along the relaxation cycle of slow system (2), the $y$-nullcline of fast system (1), while $X$ remains in phase I of the relaxation cycle, will be at $x=a<1$. Then, if $a>1-\sqrt{\delta}$, although a priori the limit cycle $\gamma_{\boldsymbol{\mu}}$ could display small oscillations, the phase plane of the fast system at the beginning of phase I is as represented in Fig. 5 and the orbits entering the extra zone from the right zone are not able to display small oscillations; they pass immediately to the center zone because they are attracted by the relaxation oscillation cycle of the fast system, and the pulses begin. As we can approximate $X_{\min } \simeq-2 k_{2}-1$, for $\varepsilon$ small enough (see (4)) conditions (7) and (6) are equivalent, concluding the proof.

Let us denote

$$
c_{\min }=\frac{a_{2}+1}{2 k_{2}+1} .
$$

If we take $c>c_{\min }$, the system may present some small oscillations before the pulsatile regime. This is due to the fact that, in this case, at the beginning of phase I, the phase portrait of the fast system is as shown in Fig. 7 and the orbits entering the extra zone from the right zone can be in the basin of attraction of the linear center, and display some small oscillations before they enter the center zone. We also denote

$$
c_{\max }=\frac{a_{2}+1+\sqrt{\delta}}{2 k_{2}+1},
$$

which corresponds to the value of $c$ such that the vertical nullcline is on the separation line $x=1+\sqrt{\delta}$. Note that, for $c>c_{\max }$, the flow passes through the whole extra zone, see Fig. 9. As we argue below, this configuration gives rise to the maximal number of small oscillations. Now, we wonder about the exact number of small oscillations exhibited by the system, depending on the parameters. To do the computation, we first reduce the $4 \mathrm{D}$ system to a three-dimensional one during the pause and pulsatile regime. 


\subsection{Three-dimensional reduction with three time scales during the pause and} pulsatile regime

During phase I of the limit cycle of the forcing system (2), variables $X$ and $Y$ follow the slowest time scale and the current $(X, Y)$ point remains in an $O(\varepsilon)$ neighborhood of the $X$-nullcline. This reads $Y=h_{\varepsilon}(X)$, where $(X, \varepsilon) \mapsto h_{\varepsilon}(X)$ is an analytic function on $(-\infty,-1) \times \mathbb{R}_{+}^{*}$ and $h_{0}=g$. Therefore, on $(-\infty,-1), h_{\varepsilon}^{\prime}(X)=g^{\prime}(X)+O(\varepsilon)=-1+O(\varepsilon)$.

We introduce a reduced system obtained from (1)-(2) with $f(x)$ given in (3), assuming that $Y=h_{\varepsilon}(X)$. We differentiate this condition, with $\varepsilon$ constant, $\dot{Y}=\dot{X} h_{\varepsilon}^{\prime}(X)$. By replacing the dynamics of $\dot{Y}$ in(1)-(2), one obtains the following three-dimensional system with three different time scales,

$$
\left\{\begin{array}{l}
\dot{x}=-y+f(x) \\
\dot{y}=\delta\left(x+a_{2}+c X\right) \\
\dot{X}=\varepsilon \delta\left(\frac{X+b_{1}\left(-X-k_{2}-1+O(\varepsilon)\right)+b_{2}}{-1+O(\varepsilon)}\right) .
\end{array}\right.
$$

Between $c_{\min }$ and $c_{\max }$, there exist intermediate values of $c=c_{n}$, for which the attractive limit cycle $\gamma_{\boldsymbol{\mu}}$ of system (1)-(2) switches from $n$ to $n+1$ small oscillations, until some maximum value $n$ to be computed. In the next subsection, we approximate these $c_{n}$ values, which correspond to the moments when the orbit passes through the primary and the secondary canards, using the reduced system (8).

2.3. Number of small oscillations. Passage through primary and secondary canards

In the previous subsection, we have computed two extreme values of $c$ : $c_{\text {min }}$, such that for $c<c_{\text {min }}$ the limit cycle $\gamma_{\boldsymbol{\mu}}$ does not display any small oscillation, and $c_{\max }$, such that for $c>c_{\max }$, the flow covers the whole extra zone, giving rise to the maximum number of small oscillations. Now, we aim to find the values of $c$ such that the system passes from $n$ to $n+1$ small oscillations, until reaching the maximum number. As a first step in the computation, we define the Fenichel slow manifolds of the reduced system (8). In the following we consider $b_{1} \neq 1$. The case $b_{1}=1$ can be analyzed analogously.

Proposition 2. Consider the three-dimensional system (8) with $\varepsilon$ and $\delta$ small enough, under hypotheses (H1), (H2) and $b_{1} \neq 1$. A canonical choice 
for the attracting and repelling Fenichel slow manifolds is given by the planes

$$
\begin{aligned}
& S_{a, \delta} \equiv \\
& \left\{x+a_{2}+c \hat{X}-\frac{\lambda_{s}^{R}}{\delta}\left(y-2-a_{2}-c \hat{X}\right)+\frac{c\left(2 \delta+(\sqrt{1-4 \delta}-1)\left(\lambda_{s s}+1\right)\right)}{2\left(\delta+\lambda_{s s}\left(\lambda_{s s}+1\right)\right)}(X-\hat{X})=0\right\}
\end{aligned}
$$

and

$$
\begin{aligned}
& S_{r, \delta} \equiv \\
& \left\{x+a_{2}+c \hat{X}-\frac{\lambda_{s}^{C}}{\delta}\left(y+a_{2}+c \hat{X}\right)+\frac{c\left(2 \delta-(\sqrt{1-4 \delta}-1)\left(\lambda_{s s}-1\right)\right)}{2\left(\delta+\lambda_{s s}\left(\lambda_{s s}-1\right)\right)}(X-\hat{X})=0\right\}
\end{aligned}
$$

respectively, where

$$
\hat{X}=\frac{b_{2}-b_{1}\left(1+k_{2}\right)}{b_{1}-1}
$$

and

$$
\lambda_{s s}=\left(b_{1}-1\right) \varepsilon \delta .
$$

Proof. Consider the three-dimensional system (8). In each linearity zone, the eigenvalue corresponding to the super-slow direction is $\lambda_{s s}$ given in (12). Let us study the existence of invariant planes of the system, depending on the intermediate and fast directions.

In the central zone $x \in(-1,1-\sqrt{\delta})$, the eigenvalues corresponding to the fast and intermediate directions are given by

$$
\lambda_{f}^{C}=\frac{1}{2}(1+\sqrt{1-4 \delta}) \quad \text { and } \quad \lambda_{s}^{C}=\frac{1}{2}(1-\sqrt{1-4 \delta})
$$

and are positive for $\delta$ small enough. There exists an invariant plane corresponding to the intermediate and slow directions,

$$
\begin{aligned}
& \Pi_{r} \equiv \\
& \left\{x+a_{2}+c \hat{X}-\frac{\lambda_{s}^{C}}{\delta}\left(y+a_{2}+c \hat{X}\right)+\frac{c\left(2 \delta-(\sqrt{1-4 \delta}-1)\left(\lambda_{s s}-1\right)\right)}{2\left(\delta+\lambda_{s s}\left(\lambda_{s s}-1\right)\right)}(X-\hat{X})=0\right\}
\end{aligned}
$$

where $\hat{X}$ is given in (11), and it is repelling in the fast direction. Moreover, when $\delta \rightarrow 0$, it tends to the critical manifold $y=x$. It follows that $\Pi_{r}$ provides a canonical choice of a repelling Fenichel slow manifold $S_{r, \delta}[10,11,16,17]$.

In the right zone $x>1+\sqrt{\delta}$, the eigenvalues corresponding to the fast and intermediate directions are

$$
\lambda_{f}^{R}=-\frac{1}{2}(1+\sqrt{1-4 \delta}) \quad \text { and } \quad \lambda_{s}^{R}=-\frac{1}{2}(1-\sqrt{1-4 \delta})
$$


and are negative for $\delta$ small enough. There exists an invariant plane corresponding to the intermediate and slow directions,

$$
\begin{aligned}
& \Pi_{a} \equiv \\
& \left\{x+a_{2}+c \hat{X}-\frac{\lambda_{s}^{R}}{\delta}\left(y-2-a_{2}-c \hat{X}\right)+\frac{c\left(2 \delta+(\sqrt{1-4 \delta}-1)\left(\lambda_{s s}+1\right)\right)}{2\left(\delta+\lambda_{s s}\left(\lambda_{s s}+1\right)\right)}(X-\hat{X})=0\right\}
\end{aligned}
$$

and it is attracting in the fast direction. Moreover, when $\delta \rightarrow 0$, it tends to the critical manifold $y=-x+2$. It follows that $\Pi_{a}$ provides a canonical choice of an attracting Fenichel slow manifold $S_{a, \delta}[10,11,16,17]$, finishing the proof.

Now that we have defined the attracting and repelling Fenichel slow manifolds for system (8), it is possible to define the primary and secondary canards, as follows.

Definition 1. Consider system (8) with $\varepsilon$ and $\delta$ small enough, under hypotheses (H1), (H2) and $b_{1} \neq 1$. We call canard a connection between the attracting and repelling Fenichel slow manifolds $S_{a, \delta}$ and $S_{r, \delta}$ defined in (9) and (10), respectively, through the flow in the extra zone $x \in(1-\sqrt{\delta}, 1+\sqrt{\delta})$. If the connection is such that the flow does not make any loop, it is called the primary canard. If during the connection the flow makes $n$ loops, it is called the $n^{\text {th }}-$ secondary canard.

It is worth noticing that due to the presence of two slow variables, the primary and secondary canards of system (8) are robust (they persist under small parameter variation). This is the case of smooth systems [8, 19] and has already been observed in [9]. However, it is not the aim of this work to find their general expressions. Rather, we intend to compute the parameter values of system (1)-(2) such that the current orbit crosses the primary or a secondary canard and contains canard segments that are responsible for the adding of one small oscillation. In particular, we will focus our attention on the limit cycle $\gamma_{\boldsymbol{\mu}}$ whose existence has been established in Theorem 1, although the proof is analogous for every orbit passing through the extra zone close to the fast nullcline, no matter if the orbit is globally cyclic or not. In the next theorem, we give an approximation of the values of $c$, depending on the other parameters of the system, for which the limit cycle $\gamma_{\boldsymbol{\mu}}$ goes through the primary or a secondary canard. An instance of this situation is represented in Figure 10. 
Theorem 2. Consider system (1)-(2), with $\varepsilon$ and $\delta$ small enough, under hypotheses (H1), (H2) and $b_{1} \neq 1$. The values for which a segment of the limit cycle $\gamma_{\boldsymbol{\mu}}$ is included in $S_{r, \delta}$ and passes very close to the primary or the $n^{\text {th }}$-secondary canard, are given by

$$
\begin{aligned}
& c_{n}\left(X_{\min }\right)=-\frac{a_{2}+1}{X_{\min }}-\frac{\left(\left(a_{2}+1\right) \varepsilon\left(b_{2}+X_{\min }-b_{1}\left(k_{2}+X_{\min }+1\right)\right)+X_{\min }\right) \sqrt{\delta}}{X_{\min }^{2}} \\
& +\frac{\left(\varepsilon\left(\pi\left(a_{2}+1\right)(4 n+1)-2 a_{2}\right)\left(b_{1}\left(k_{2}+X_{\min }+1\right)-b_{2}-X_{\min }\right)+4 X_{\min }\right) \delta}{2 X_{\min }^{2}} \\
& +O\left(\delta^{3 / 2}\right),
\end{aligned}
$$

where $n \in \mathbb{Z} \cap\left[0, r_{2}\right)$, with

$$
\begin{aligned}
& r_{2}=\frac{1}{4 \pi} . \\
& \left(\frac{4 X_{\min }\left(2 a_{2} \sqrt{\delta}+a_{2}-(\sqrt{1-4 \delta}-2) \sqrt{\delta}+1\right)}{\varepsilon\left(2\left(a_{2}+1\right)^{2} \sqrt{\delta}+\left(a_{2}+1\right)^{2}+2 \delta^{3 / 2}-\delta\right)\left(-b_{1}\left(k_{2}+X_{\min }+1\right)+b_{2}+X_{\min }\right)}\right. \\
& \left.+\frac{\sqrt{1-4 \delta}-(2+\pi) \sqrt{\delta}-3}{\sqrt{\delta}}\right)+O\left(\varepsilon^{2}\right),
\end{aligned}
$$

and $X_{\min }$ corresponds to the minimal value reached by variable $X$ along the relaxation cycle of the forcing system (2), approximated by (4). The value $c_{0}$ corresponds to the passage through the primary canard, and each other $c_{n}$ value to the $n$-secondary canard.

Proof. We aim to compute the parameter values such that the limit cycle $\gamma_{\boldsymbol{\mu}}$ connects the attracting and repelling Fenichel slow manifolds $S_{a, \delta}$ and $S_{r, \delta}$ through the extra zone $x \in(1-\sqrt{\delta}, 1+\sqrt{\delta})$. In the extra zone, the eigenvalues of the fast and intermediate directions are $\lambda= \pm \mathrm{i} \sqrt{\delta}$, corresponding to a two dimensional linear center, therefore the orbits behave in an oscillatory manner.

Note that, when the forcing system (2) is in the relaxation oscillation regime (hypothesis (H1)), the forced system (1) always enters the extra zone while $X$ is in phase I. Also, given that plane $S_{a, \delta}$ attracts the flow in the fast direction, we can assume that the entry point is located on $S_{a, \delta}$.

While looking for the orbits that connect to $S_{r, \delta}$, we search for the orbits in the extra zone whose initial condition is at the intersection between 
the separation boundary $x=1+\sqrt{\delta}$ and $S_{a, \delta}$, and end up arriving at the separation boundary $x=1-\sqrt{\delta}$ on the repelling manifold $S_{r, \delta}$.

By taking $x=1+\sqrt{\delta}$ in (15) we can find $y_{a}=y\left(X_{a}\right)$, where $X_{a}$ is defined in (5) and $a \in(1-\sqrt{\delta}, 1+\sqrt{\delta})$, such that the line of initial conditions in $S_{a, \delta}$ intersecting the separation plane $(x=1+\sqrt{\delta})$, parameterized by $X_{a}$, is given by

$$
\mathbf{p}_{a}=\left(1+\sqrt{\delta}, y_{a}, X_{a}\right) .
$$

Let us denote

$$
\mathbf{x}^{E}\left(t ; \boldsymbol{\mu}, \mathbf{p}_{0}\right)=\left(x^{E}\left(t ; \boldsymbol{\mu}, \mathbf{p}_{0}\right), y^{E}\left(t ; \boldsymbol{\mu}, \mathbf{p}_{0}\right), X^{E}\left(t ; \boldsymbol{\mu}, \mathbf{p}_{0}\right)\right)^{T}
$$

the solution of system (8) in the extra zone with $\boldsymbol{\mu}=\left(\varepsilon, \delta, b_{1}, b_{2}, a_{2}, k_{2}, c\right)$, namely

$$
\left\{\begin{array}{l}
\dot{x}=-y+1-\sqrt{\delta}, \\
\dot{y}=\delta\left(x+a_{2}+c X\right), \\
\dot{X}=\varepsilon \delta\left(X\left(b_{1}-1\right)+b_{1}\left(k_{2}+1\right)-b_{2}\right),
\end{array}\right.
$$

and initial condition $\mathbf{p}_{0}$. We are looking for the set of parameters satisfying the following conditions

$$
\left\{\begin{array}{l}
x^{E}\left(s ; \boldsymbol{\mu}, \mathbf{p}_{a}\right)=1-\sqrt{\delta}, \\
\left(\left(y^{E}\left(s ; \boldsymbol{\mu}, \mathbf{p}_{a}\right), X^{E}\left(s ; \boldsymbol{\mu}, \mathbf{p}_{a}\right)\right) \in\left\{S_{r, \delta} \cap x=1-\sqrt{\delta}\right\},\right. \\
x^{E}\left(t ; \boldsymbol{\mu}, \mathbf{p}_{a}\right) \in(1-\sqrt{\delta}, 1+\sqrt{\delta}), \quad \forall t \in(0, s),
\end{array}\right.
$$

for some $t=s>0$.

First, we look for the value of $s$ such that the first equation of (19) is satisfied. To approximate this value, we solve system (18) in $x, y$, taking $\varepsilon=0$, hence assuming $X=X_{a}$. We obtain:

$$
\begin{aligned}
& x_{\{\varepsilon=0\}}^{E}:=\left.x^{E}\left(s ; \boldsymbol{\mu}, \mathbf{p}_{a}\right)\right|_{\varepsilon=0}= \\
& -a_{2}-c X_{a}+\left(a_{2}+c X_{a}+1+\sqrt{\delta}\right) \cos (\sqrt{\delta} t)+\frac{1-\sqrt{\delta}-y_{a}}{\sqrt{\delta}} \sin (\sqrt{\delta} t) .
\end{aligned}
$$

With this expression of the solution $x^{E}$, we compute, from the first equation of (19)

$$
s=\frac{1}{\sqrt{\delta}}\left(\frac{\pi}{2}+2 \pi n+O(\sqrt{\delta})\right),
$$


with $n \in \mathbb{N}$.

Note that the third equation of system (18) is independent, which makes it possible to compute the solution,

$$
X^{E}\left(t ; \boldsymbol{\mu}, \mathbf{p}_{a}\right)=X_{a}+\varepsilon \delta t\left(b_{1}\left(1+k_{2}+X_{a}\right)-b_{2}-X_{a}\right)+O\left(\varepsilon^{2}\right) .
$$

With the approximation up to order $\varepsilon$ of the solution in $X$, we solve system (18) in $x, y$, and obtain,

$$
\begin{aligned}
x^{E}\left(t ; \boldsymbol{\mu}, \mathbf{p}_{a}\right)= & x_{\{\varepsilon=0\}}^{E}+c \sqrt{\delta} \varepsilon\left(b_{2}+X_{a}-b_{1}\left(1+k_{2}+X_{a}\right)\right)(\sqrt{\delta} t-\sin (\sqrt{\delta} t)), \\
y^{E}\left(t ; \boldsymbol{\mu}, \mathbf{p}_{a}\right)= & y_{a} \cos (\sqrt{\delta} t)+(1-\cos (\sqrt{\delta} t)) . \\
& \left(1-\sqrt{\delta}\left(1+\varepsilon \sqrt{\delta} c\left(b_{2}+X_{a}-b_{1}\left(1+k_{2}+X_{a}\right)\right)\right)\right)+ \\
& \sqrt{\delta}\left(a_{2}+1+\sqrt{\delta}+c X_{a}\right) \sin (\sqrt{\delta} t),
\end{aligned}
$$

where $x_{\{\varepsilon=0\}}^{E}$ is defined in (20). Now, from the equation of $S_{r, \delta}$ in (14), the second condition in (19) is written as

$$
\begin{aligned}
& 1-\sqrt{\delta}+a_{2}+c \hat{X}-\frac{\lambda_{s}^{C}}{\delta}\left(y^{E}\left(s ; \boldsymbol{\mu}, \mathbf{p}_{a}\right)+a_{2}+c \hat{X}\right)+ \\
& \frac{c\left(2 \delta-(\sqrt{1-4 \delta}-1)\left(\lambda_{s s}-1\right)\right)}{2\left(\delta+\lambda_{s s}\left(\lambda_{s s}-1\right)\right)}\left(X^{E}\left(s ; \boldsymbol{\mu}, \mathbf{p}_{a}\right)-\hat{X}\right)=0
\end{aligned}
$$

with $s$ given in (21). From this equation, taking into account that the value of $X$ for which the flow of fast system (1) enters the extra zone can be approximated by $X_{\min }$, (beginning of phase $\mathrm{I}$ ), we find $c_{n}\left(X_{\min }\right)$ given in (16). However, not every $n \geq 0$ corresponds to an orbit connecting to $S_{r, \delta}$. We need, in addition, that inequalities in (19) (third condition) be satisfied.

Consider the first part of the inequality in the last line of (19), that is,

$$
x^{E}\left(t ; \boldsymbol{\mu}, \mathbf{p}_{a}\right)>1-\sqrt{\delta}, \quad \forall t \in(0, s) .
$$

Function $h(t):=x^{E}\left(t ; \boldsymbol{\mu}, \mathbf{p}_{a}\right)-1+\sqrt{\delta}$ is the sum of a linear function in $t$ and an oscillatory function in $\sin (\sqrt{\delta} t)$ and $\cos (\sqrt{\delta} t)$. The slope of the linear function is

$$
c \varepsilon \delta\left(b_{2}+X_{a}-b_{1}\left(1+k_{2}+X_{a}\right)\right)=-c \frac{\partial X^{E}}{\partial t}\left(0 ; \boldsymbol{\mu}, \mathbf{p}_{a}\right)<0,
$$

as $X$ is increasing during phase I. On the other hand, $h(s)=0,(s$ is the 
endpoint of the interval, see (22)). Consider the derivative at $t=s$,

$$
\dot{h}(s)=\frac{\partial x^{E}}{\partial t}\left(s ; \boldsymbol{\mu}, \mathbf{p}_{a}\right)=-y^{E}\left(s ; \boldsymbol{\mu}, \mathbf{p}_{a}\right)+1-\sqrt{\delta} .
$$

One can see that for $\delta$ small enough, function $y^{E}\left(s ; \boldsymbol{\mu}, \mathbf{p}_{a}\right)$ is decreasing with respect to $n$, hence, $-y^{E}\left(s ; \boldsymbol{\mu}, \mathbf{p}_{a}\right)$ is increasing. Starting from the first $n$ for which $-y^{E}\left(s ; \boldsymbol{\mu}, \mathbf{p}_{a}\right)+1-\sqrt{\delta}$ is positive, the $c_{n}$ values do not correspond to secondary canards, as function $h(t)$ becomes negative before $t=s$. It is possible to compute the root of $-y^{E}\left(s ; \boldsymbol{\mu}, \mathbf{p}_{a}\right)+1-\sqrt{\delta}=0$, which is

$$
\begin{aligned}
r_{1}= & \left(1-\frac{\sqrt{\delta}}{1+a_{2}}+\frac{\delta}{\left(1+a_{2}\right)^{2}}\right) . \\
& \frac{X_{a}}{\varepsilon \pi\left(1+a_{2}\right)\left(b_{2}+X_{a}-b_{1}\left(1+k_{2}+X_{a}\right)\right)}+O\left(\delta^{3 / 2}\right),
\end{aligned}
$$

and it follows that inequality (22) is satisfied for $n \leq\left\lfloor r_{1}\right\rfloor$.

Consider the second part of the inequality in the last line of (19), that is,

$$
x^{E}\left(t ; \boldsymbol{\mu}, \mathbf{p}_{a}\right)<1+\sqrt{\delta}, \quad \forall t \in(0, s) .
$$

Function $m(t):=-x^{E}\left(t ; \boldsymbol{\mu}, \mathbf{p}_{a}\right)+1+\sqrt{\delta}$ is again the sum of a linear function in $t$ and an oscillatory function in $\sin (\sqrt{\delta} t)$ and $\cos (\sqrt{\delta} t)$. The slope of the linear function is

$$
-c \varepsilon \delta\left(b_{2}+X_{a}-b_{1}\left(1+k_{2}+X_{a}\right)\right)=c \frac{\partial X^{E}}{\partial t}\left(0 ; \boldsymbol{\mu}, \mathbf{p}_{a}\right)>0,
$$

as $X$ is increasing during phase I. On the other hand, $m(0)=0$. Consider the derivative at $t=0$,

$$
\dot{m}(s)=-\frac{\partial x^{E}}{\partial t}\left(0 ; \boldsymbol{\mu}, \mathbf{p}_{a}\right)=y^{E}\left(0 ; \boldsymbol{\mu}, \mathbf{p}_{a}\right)-1+\sqrt{\delta}=y_{a}-1+\sqrt{\delta} .
$$

One can check that for $\delta$ small enough, function $y_{d}$ is decreasing with respect to $n$. From the first $n$ such that $y_{a}-1+\sqrt{\delta}$ is negative, the $c_{n}$ values do not correspond to secondary canards, as function $m(t)$ becomes negative before $t=s$. We can compute the root $r_{2}$ of $y_{a}-1+\sqrt{\delta}=0$, given in (17) and obtain that inequality (23) is satisfied for $n \leq\left\lfloor r_{2}\right\rfloor$.

Finally, $r_{1}-r_{2}=1 /(2 \pi \sqrt{\delta})+1 / \pi+O(\sqrt{\delta})$, hence $r_{1}>r_{2}$ for $\delta$ small. As 
we need both inequalities to be satisfied, the maximum number of $n$ is $\left\lfloor r_{2}\right\rfloor$, which finishes the proof.

In the sequence of figures 1, 11 and 12, we can observe the loss of one small oscillation due to the passage of the limit cycle through a secondary canard. The limit cycle starts with 5 small oscillations in Fig. 1 and ends up with 4 in Fig. 12. In Fig. 11, the canard-induced transition is taking place, which gives birth to an intermediate oscillation between the pause and pulsatile regime, that one can distinguish on the figure. In this case, we have also represented the phase plane in variables $x, y$, in Fig. 10. The parameter values are $a_{2}=2, b_{1}=0, b_{2}=0.5, k_{2}=0.6, \varepsilon=0.025, \delta=0.2$ and $c=1.5$ in Fig. $1, c=1.4951$ in Fig. 11 and $c=1.49$ in Fig. 12 .

A direct consequence of Theorem 2 is that we can control the number of small oscillations of limit cycle $\gamma_{\boldsymbol{\mu}}$ for a given set of parameters.

Corollary 1. Consider system (1)-(2) under hypotheses (H1), (H2) and $b_{1} \neq 1$. Fix all parameters of the system except $c$.

1. If $c \in\left(c_{n}, c_{n+1}\right)$, where $c_{n}$ is given in (16), the number of small oscillations of the limit cycle equals $n+1$.

2. The maximum number of small oscillations that the limit cycle $\gamma_{\boldsymbol{\mu}}$ can exhibit is approximated by the smallest integer larger than $r_{2}$ as defined in (17).

\section{Addition-subtraction of small oscillations and pulses}

In section 2, we have computed the number of small oscillations of limit cycle $\gamma_{\boldsymbol{\mu}}$ of system (1)-(2), depending on the parameters of the system. In Corollary 1, after fixing all the parameters of the system except $c$, we give a characterization of that number depending on the value of the regular parameter $c$. In particular, decreasing the value of $c$ leads to a subtraction in the number of small oscillations for $c=c_{n}$ given in (16). In this section, we now tackle the question of whether such a subtraction of small oscillations is compensated for by the adding of one pulse just before the surge, or the number of pulses is unchanged. Note that, if a pulse adding occurs, it is due to the (lower) canard superexplosion that is responsible for the creation of a new relaxation oscillation of the fast system. 
We know from Theorem 1 that the period of limit cycle $\gamma_{\boldsymbol{\mu}}$, corresponds to the period of the relaxation cycle of the forcing system (2), which does not depend on parameter $c$. Then, the main question to address here is whether, during the time that the limit cycle loses in the small oscillations regime when $c$ decreases, the lower canard cycle is able to create a new pulse, or not.

In the next proposition, we compute the period of one small oscillation and we approximate the period of one pulse.

Proposition 3. Consider limit cycle $\gamma_{\boldsymbol{\mu}}$ of system (1)-(2). The following statements hold.

1. The period of one small oscillation is given by

$$
T_{s}=2 \pi / \sqrt{\delta}
$$

2. The period of one pulse is approximated by

$$
T_{p}=-\frac{\log \left(\frac{p^{2}-1}{p^{2}-9}\right)}{\delta},
$$

where $p \in(-1,1-\sqrt{\delta})$ is the first component of the equilibrium point of fast system (1).

Proof. The first statement comes from the fact that, in the extra zone of system (1), the linear configuration type is a center with period $2 \pi / \sqrt{\delta}$.

For the second statement, we just have to consider expression (3.7) of [12], and perform the change of variables (3.8) with $a_{1}=1, a_{1}=0$ and $k_{1}=1$.

From Proposition 3, it follows that the number of small oscillations that has to be lost in order to win one extra pulse, depends on $\delta$. More precisely, the time needed to display one small oscillation is of order $1 / \sqrt{\delta}$, while the time needed to display one pulse is of order $1 / \delta$. Therefore, when $\delta$ decreases, the number of small oscillations that need to be lost in order to add one extra pulse increases, as we establish in the next theorem.

Theorem 3. Consider system (1)-(2) under hypotheses (H1), (H2) and $b_{1} \neq$ 1. Fix all parameters of the system except $c$. Assume that $r_{2}>0$, where $r_{2}$ 
is defined in (17). While decreasing $c$, there may exist two different types of $c_{n}$ values given in (16), for which the number of small oscillations exhibited by the limit cycle is depleted by one, namely,

1. $c_{n}=c_{n}^{0}$, the number of pulses remains the same after the passage through $c_{n}^{0}$ while decreasing $c$.

2. $c_{n}=c_{n}^{1}$, one extra pulse appears after the passage through $c_{n}^{1}$ while decreasing $c$, to compensate for the decrease in the duration covered by small oscillations.

The number of $c_{n}^{0}$ values that we find between two $c_{n}^{1}$ depends on $\delta$ and increases while $\delta$ decreases. Moreover, in an exponentially small neighborhood of $c_{n}$, as the system goes through the canard explosion, one pulse disappears at the end of the pulsatility phase and another is created from a small oscillation at the beginning of the pulsatility phase.

Proof. The fact that, in both cases, in an exponentially small neighborhood of $c_{n}$, one pulse disappears at the end of the pulsatility phase and another is created from a small oscillation at the beginning of the pulsatility phase, is due to the (upper) canard explosion. In particular, in the canard transition from one small oscillation to one pulse (see Fig. 11), it takes the orbit a time $O(-\log (\delta) / \delta)$ to perform the transition (see $T^{L}$ in [12], change the length of the extra piece to $2 \sqrt{\delta}$ and take $h=1)$.

The remaining follows from Proposition 3 and the fact that the period of limit cycle $\gamma_{\boldsymbol{\mu}}$ of system (1)-(2) does not depend on $c$.

In the situation represented in figures 1,11 and $12, \delta=0.2$ and the loss of one small oscillation is compensated for by one extra pulse. By decreasing $\delta$ to 0.05 and keeping the remainder parameters unchanged, we observe that we now need to lose two small oscillations in order to add a single pulse, see figures 13,14 and 15 .

\section{Proof of Theorem 1}

The dynamics can be divided into three stages:

1. Exponentially contracting, corresponding to the passage through the surge. 
2. Exponentially expanding, corresponding to the passage near the upper fold and canard phenomenon during the transition from pulsatility to surge.

3. Neutral, corresponding to the pulsatile regime.

Let us compute the contraction-expansion rate in each stage to assess the whole contraction rate:

1. Contraction rate during the surge.

As in [13], we can consider a two-dimensional reduction of system (1)(2) with two time scales. Variable $X$ moves along phase III of the limit cycle of slow system $(2)$ and the current point $(x, y)$ follows the stable node $\mathbf{p}^{L}(X)$ lying on the left branch of $y=f(x)$. Hence, both approximations $Y \simeq g(X)$ and $y \simeq f(x)$ are valid.

By reducing the fastest time scale i.e., setting $y=f(x)$ in (1)-(2), we obtain the three dimensional system,

$$
\left\{\begin{array}{l}
(d f(x) / d x) \dot{x}=\delta\left(x+a_{2}+c X\right) \\
\dot{X}=\delta(g(X)-Y) \\
\dot{Y}=\varepsilon \delta\left(X+b_{1} Y+b_{2}\right)
\end{array}\right.
$$

Then, setting $Y=g(X)$, one gets the two-dimensional system,

$$
\left\{\begin{array}{l}
\dot{x}=\delta \frac{\left(x+a_{2}+c X\right)}{d f(x) / d x} \\
\dot{X}=\varepsilon \delta \frac{X+b_{1} g(X)+b_{2}}{d g(X) / d X} .
\end{array}\right.
$$

As time constant $\delta$ is multiplying both equations of the system, we consider the new time variable

$$
s=t \delta
$$


and the new system

$$
\left\{\begin{array}{l}
x^{\prime}=\frac{x+a_{2}+c X}{d f(x) / d x} \\
X^{\prime}=\varepsilon \frac{X+b_{1} g(X)+b_{2}}{d g(X) / d X}
\end{array}\right.
$$

where the prime denotes the derivative with respect to the new temporal variable $s$. Hence, we have obtained a two-dimensional slow-fast system with slow variable $X$ and fast variable $x$. During the surge regime, $X$ is in phase III, and then, $X>1$ so that $g(X)=-X+k_{2}+1$ and $d g(X) / d X=-1$. Regarding variable $x$, it remains along the left branch of $y=f(x)$, that is, $f(x)=-x-2$ hence, $d f(x) / d x=-1$. System (24) simplifies to

$$
\left\{\begin{array}{l}
x^{\prime}=-\left(x+a_{2}+c X\right) \\
X^{\prime}=-\varepsilon\left(X+b_{1}\left(-X+k_{2}+1\right)+b_{2}\right) .
\end{array}\right.
$$

The coefficient matrix of system (25) is given by

$$
A^{S}=\left(\begin{array}{cc}
-1 & -c \\
0 & \varepsilon\left(b_{1}-1\right)
\end{array}\right) .
$$

Note that the eigenvalue corresponding to the fast direction is -1 , so that the flow is strongly attracted towards the slow manifold. Therefore, the passage through the surge is always an exponentially strong contraction, with contraction rate $O\left(\exp \left(-C_{s} / \varepsilon\right)\right)$, where $C_{s}>0$ is a constant.

2. Contraction rate during the passage near the upper fold and canard phenomenon during the transition from pulsatility to surge.

Now, let us estimate the maximal expansion rate during the passage near the upper fold. By expansion we mean that the distance between the trajectories can grow with a certain rate forward in time. Following [12] or section 4.2 in [3], we know that the expansion rate corresponds to the time that the orbit spends in the canard transition from one small oscillation to one pulse. This time has been already computed in the proof of Theorem 3 and is $O(-\log (\delta) / \delta)$. Similarly, the maximal 
expansion for the canard phenomenon during the passage from pulsatility to surge is $O(-\log (\delta) / \delta)$. Hence, the maximal amount of expansion due to both canard phenomena is approximately $\exp (-\log (\delta) / \delta)$.

3. Contraction rate along the pulsatile regime.

The pulsatile regime corresponds to the region of transient relaxation oscillations. As the slow nullcline of (1) intersects the critical manifold in the central zone, the dynamics is purely of relaxation type, neither contracting nor expanding.

Therefore, the total contraction rate of the return map of the limit cycle is $O\left(\exp \left(-C_{s} / \varepsilon-\log (\delta) / \delta\right)\right)$, where $C_{s}>0$ is a constant. Hence, when $\varepsilon$ is small enough and for a fixed value $\delta>0$, the return map is contracting, and there exists a unique attractive limit cycle for every parameter value.

This proof is similar to the proof of Theorem 2.1 in [3].

\section{Conclusions and future work}

We have presented a 4D PWL system displaying MMOs of three different types. The system is an extension of that proposed in [13] and a PWL version of the smooth system studied in [3]. We have added one small extra piece to fast system (1) in order to account for the existence of a pause where small oscillations occur between the end of the surge and resumption of pulses, as it happen in the original smooth model [3].

We can observe that in the PWL model the small oscillations are more visible than in the smooth case, as has been previously reported in [9]. Also, the fact that the extra piece is flat makes the small oscillations more regular in comparison to those of the smooth model. A natural next step would be to change the slope of that extra piece, allowing for increasing or decreasing the size of the small oscillations. Another perspective would consist in studying the PWL equivalent of an extended, 6D version of the smooth model in which two fast subsystems are coupled together, while still being forced by the slow subsystem, leading to interesting phenomena of recurrent synchronization and desynchronization [14].

By using a singular perturbation theory approach, we have tackled three different questions. First, we have proven the existence of an attractive limit cycle of the system. Second, we have analyzed locally the passage of the limit cycle through secondary canards, resulting in a change in the number of 
small oscillations. Finally, we have studied the global compensation occurring during the passage through secondary canards with the addition-subtraction of pulses, a question that could not be tackled analytically in the smooth case due to its difficulty.

[1] F. Clément and J. P. Françoise, Mathematical Modeling of the GnRH Pulse and Surge Generator, SIAM J. Appl. Dyn. Syst., 6 (2007), pp. 441-456.

[2] F. Clément and A. Vidal, Foliation-based parameter tuning in a model of the GnRH pulse and surge generator, SIAM J. Appl. Dyn. Syst., 8 (2009), pp. 1591-1631.

[3] M. Krupa, A. Vidal, M. Desroches and F. Clément, Mixed-Mode Oscillations in a Multiple Time Scale Phantom Bursting System, SIAM J. Appl. Dyn. Syst., 11 (2012), pp. 1458-1498.

[4] M. Desroches, E. Freire, S.J. Hogan, E. Ponce, and P. Thota, Canards in piecewise-linear systems: explosions and super-explosions, Proc. R. Soc. A., 469 (2013), pp. (2154): 20120603.

[5] R. FitzHugh, Impulses and physiological states in theoretical models of nerve membrane, Biophys. J., 1 (1961), pp. 445-466.

[6] H. P. McKean, Nagumo's equation. Adv. Math., 4 (1970), pp. 209-223.

[7] J. Nagumo, S. Arimoto and S. Yoshizawa, An active pulse transmission line simulating nerve axon, Proceedings of the IRE, 50 (1962), pp. 20612070 .

[8] E. Benoit, Canards et enlacements. Publications mathématiques de 1 I.H.É.S, 72:63-91, 1990.

[9] M. Desroches, A. Guillamon, E. Ponce, R. Prohens, S. Rodrigues and A. E. Teruel, Canards, folded nodes and mixed-mode oscillations in piecewise-linear slow-fast systems. SIAM Rev., in press, 2015.

[10] N. Fenichel, Persistence and smoothness of invariant manifolds for flows, Indiana Univ. Math. J. 21(3):193-226, 1972.

[11] N. Fenichel, Geometric singular perturbation theory for ordinary differential equations, J. Differential Equations 31(1):53-98, 1979. 
[12] S. Fernández-García, M. Desroches, M. Krupa and A. E. Teruel, Canard solutions in planar piecewise linear systems with three zones, Dynam. Syst., 31(2): 173-197, 2016.

[13] S. Fernández-García, M. Desroches, M. Krupa and F. Clément, A Multiple Time Scale Coupling of Piecewise Linear Oscillators. Application to a Neuroendocrine System, SIAM J. Appl. Dyn. Syst., 14 (2015), pp. 643-673.

[14] E. Köksal Ersöz, M. Desroches, M. Krupa, and F. Clément, CanardMediated (De)Synchronization in Coupled Phantom Bursters SIAM J. Appl. Dyn. Syst., 15(1), (2016) pp. 580-608.

[15] M. Krupa, P. Szmolyan, Relaxation oscillations and canard explosion J. Diff Eq., 174: 312-368, 2001

[16] R. Prohens and A. E. Teruel, Canard trajectories in 3D piecewise linear systems, Discrete Contin. Dyn. Syst., 33: 4595-4611, 2013.

[17] R. Prohens, A. E. Teruel, and C. Vich, Slow-fast n-dimensional piecewise-linear differential systems. Preprint, 2015.

[18] H. G. Rotstein, S. Coombes and A. M. Gheorghe, Canard-like explosion of limit cycles in two-dimensional piecewise-linear models of FitzHughNagumo type, SIAM J. Appl. Dyn. Syst. 11(1): 135-180, 2012.

[19] P. Szmolyan and M. Wechselberger, Canards in $\mathbb{R}^{3}$, J. Diff. Eq., 177:419453, 2001. 


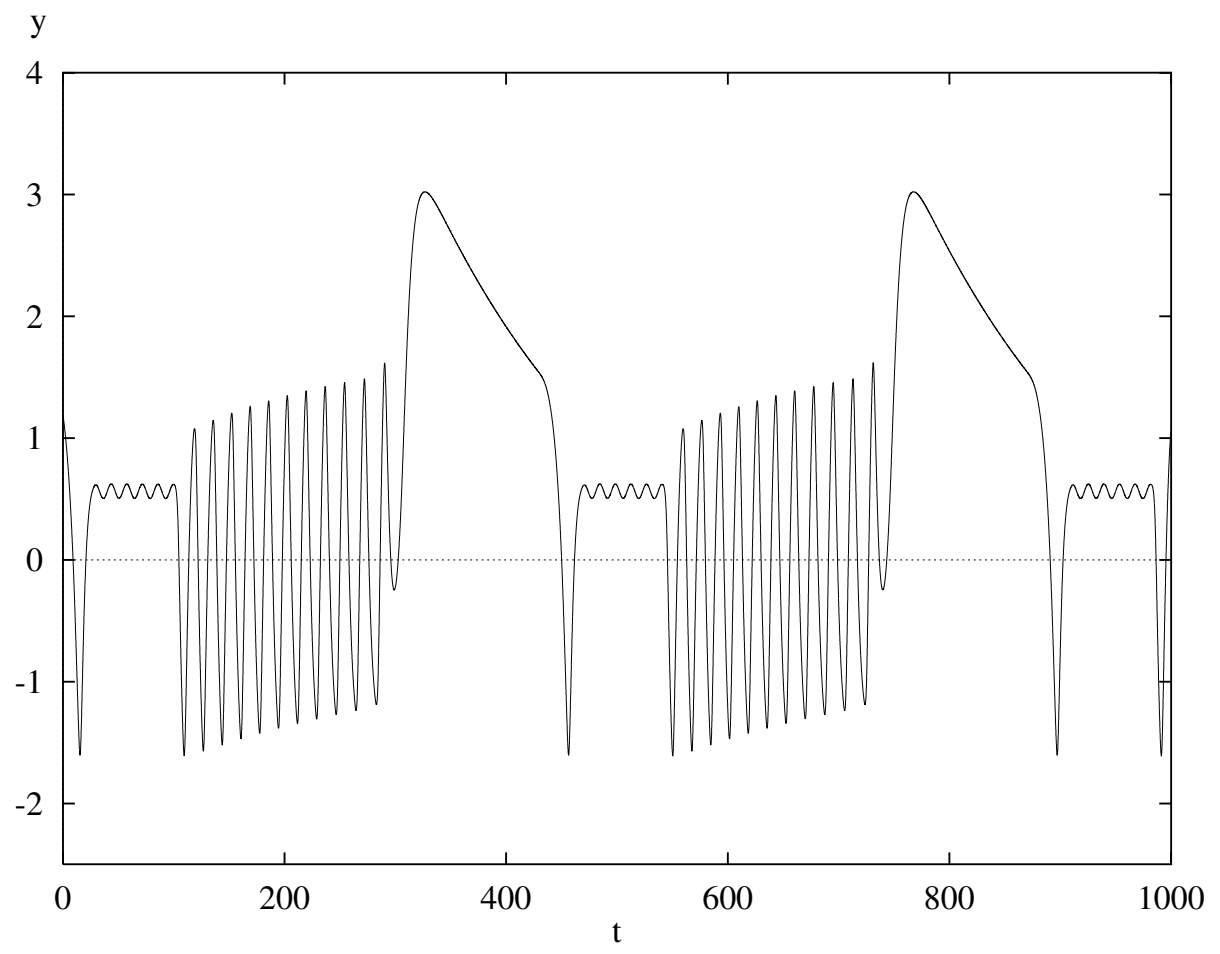

Figure 1: Second component of a typical orbit of system (1)-(2). There are three different oscillation patterns: the pulsatile regime, the surge and the pause where small oscillations takes place. The parameter values are $a_{2}=2, b_{1}=0, b_{2}=0.5, k_{2}=0.6, \varepsilon=0.025$, $\delta=0.2$ and $c=1.5$. 


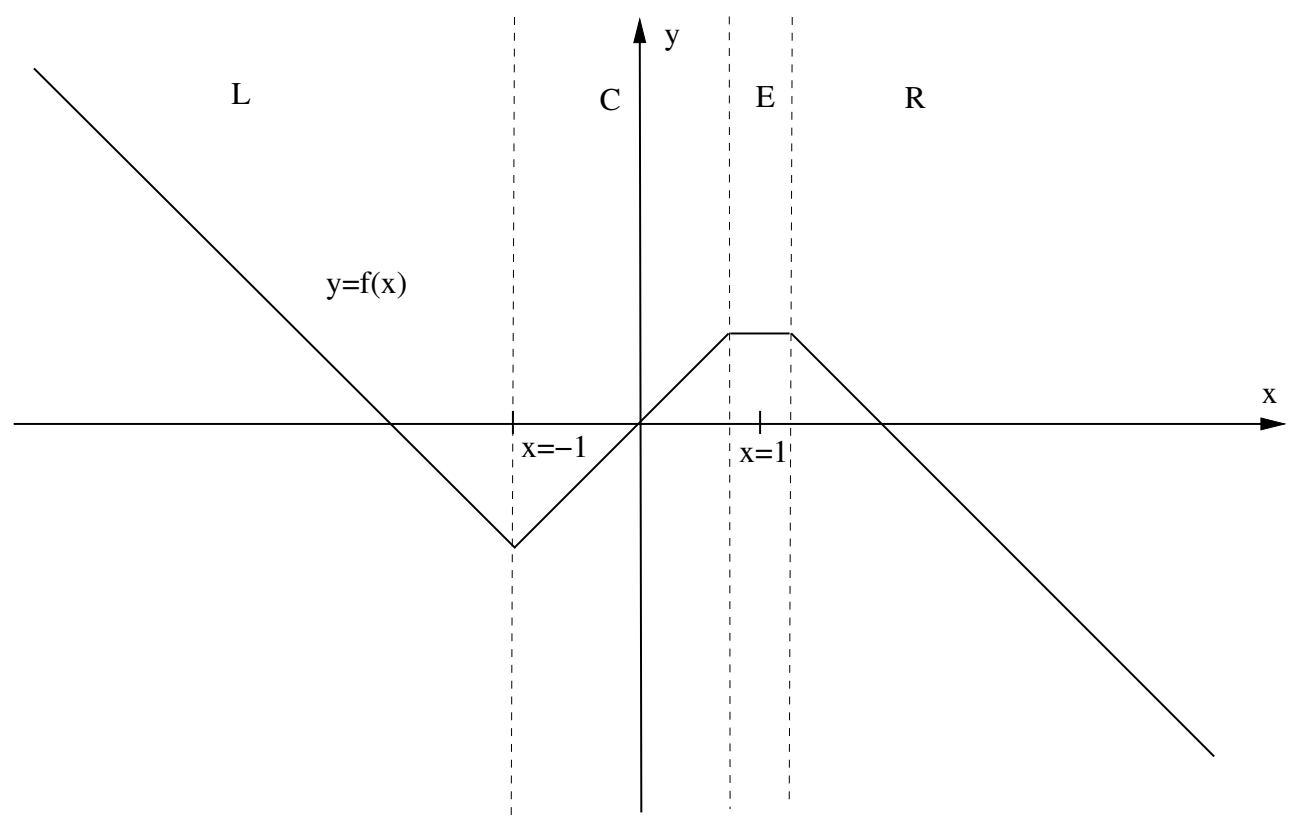

Figure 2: Sketch of the $x$-nullcline in the different zones of definition of system (1)-(2).

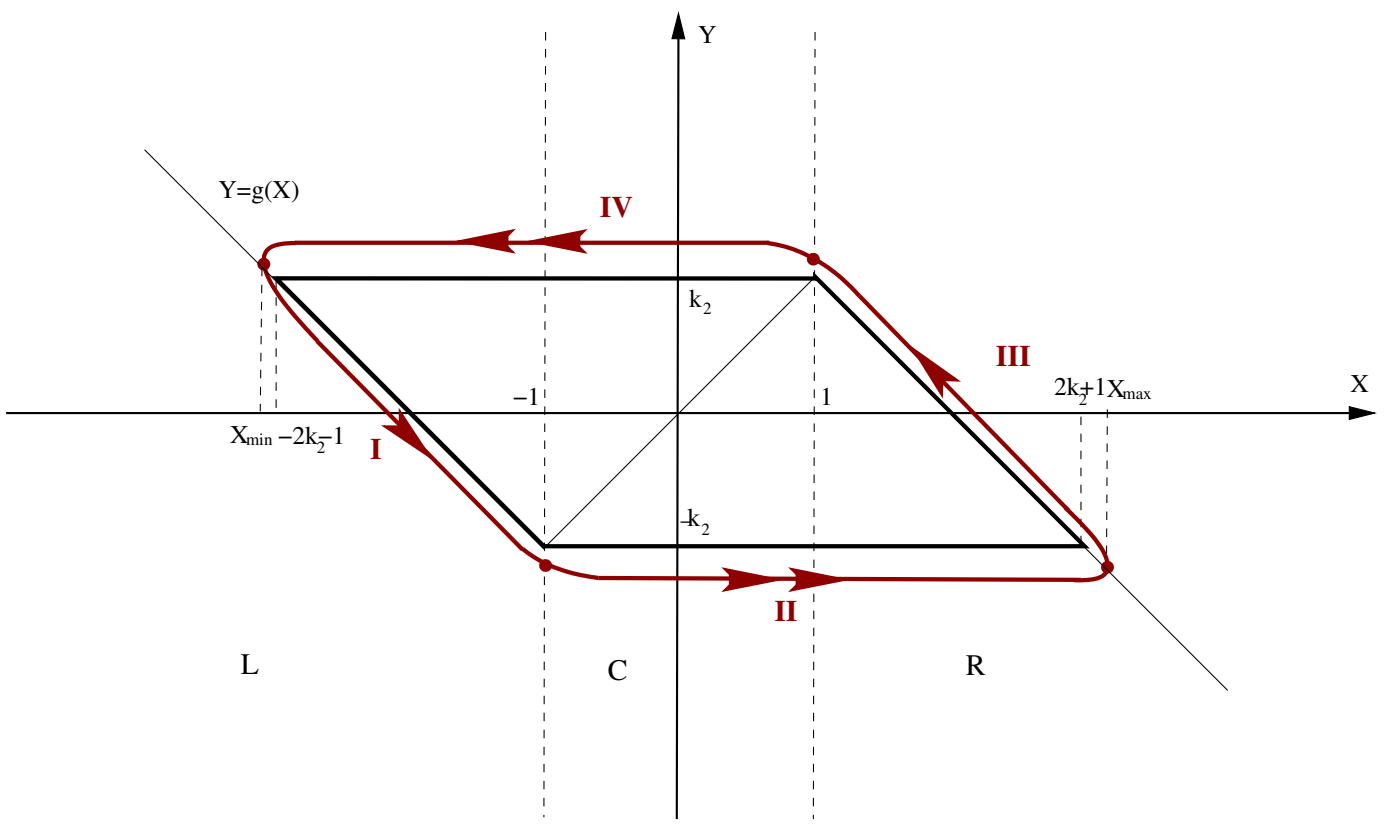

Figure 3: Sketck of the limit cycle of the forcing system (in red) and the corresponding limit set (in bold line). The path along the limit cycle is divided into four phases. 


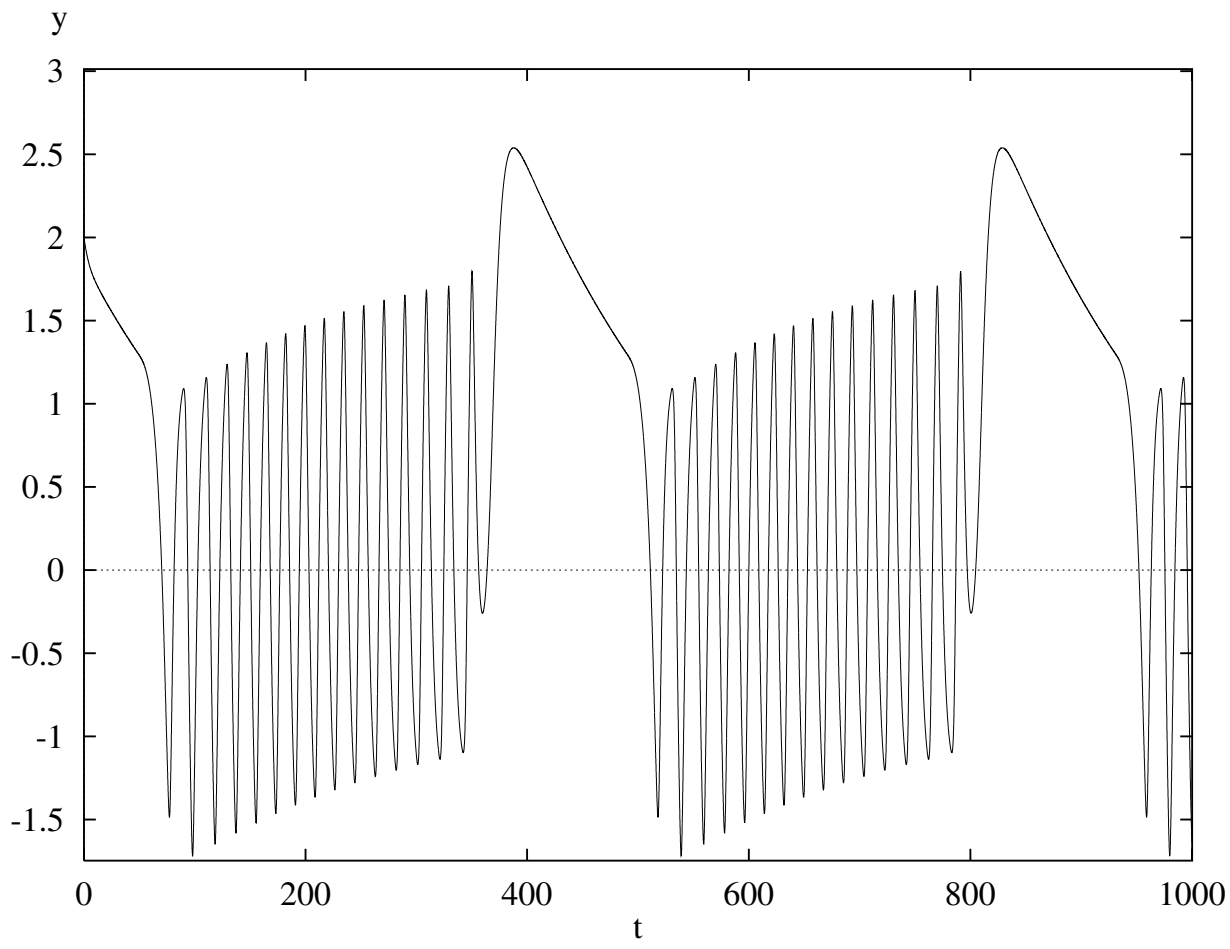

Figure 4: Second component of a typical orbit of the system studied in [13], where the parameters have been adapted to obtain the quantitative features of [3]. There are two different oscillation patterns: the pulsatile regime and the surge. 


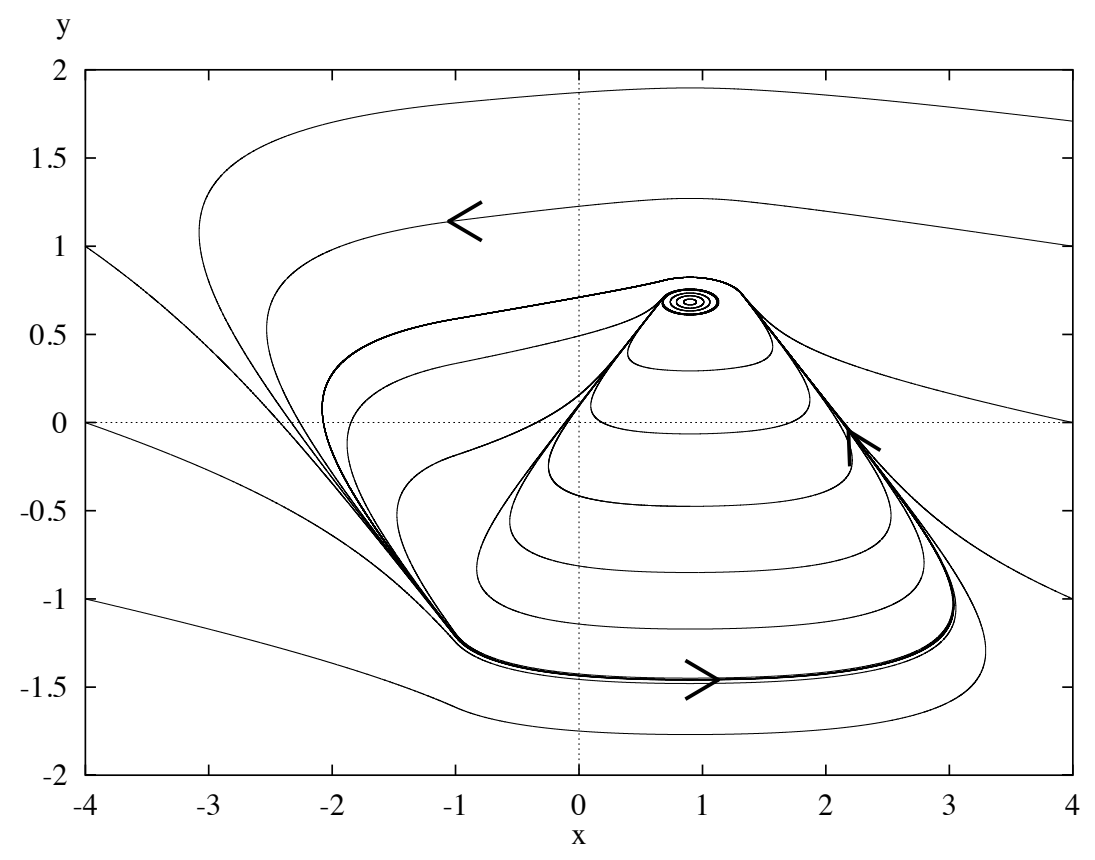

Figure 5: Phase plane of fast system (1) when the $y$-nullcline is fixed at $x=0.9$ and $\delta=0.1,(a \in(1-\sqrt{\delta}, 1))$. The system has a repulsive zonal linear center bounded by the periodic orbit tangent to the boundary $x=1-\sqrt{\delta}$, completely contained in the extra zone. There exists a relaxation limit cycle surrounding the zonal linear center. 


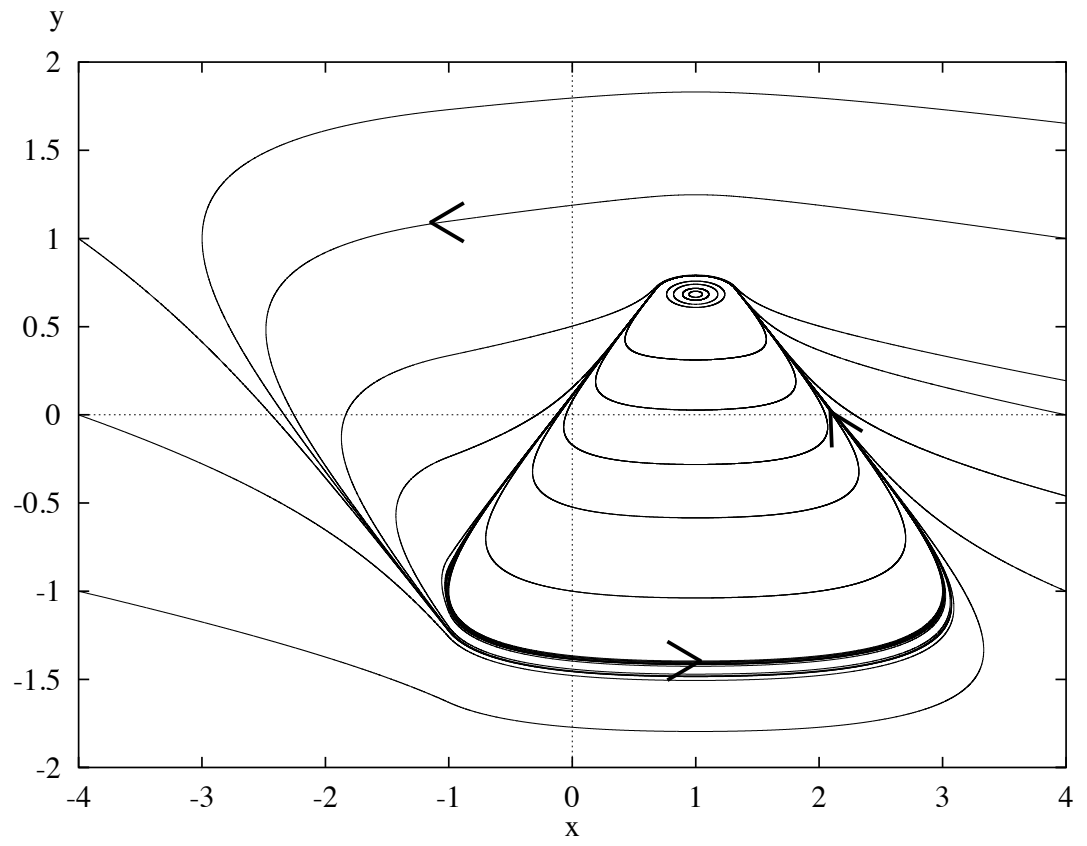

Figure 6: Phase plane of fast system (1) when the $y$-nullcline is fixed at $x=1$ and $\delta=0.1$. The system has an attractive continuum of periodic orbits surrounding the equilibrium, which in this case is located in $(x, y)=(1,1-\sqrt{\delta})$. The continuum is bounded by the maximal canard [12]. 


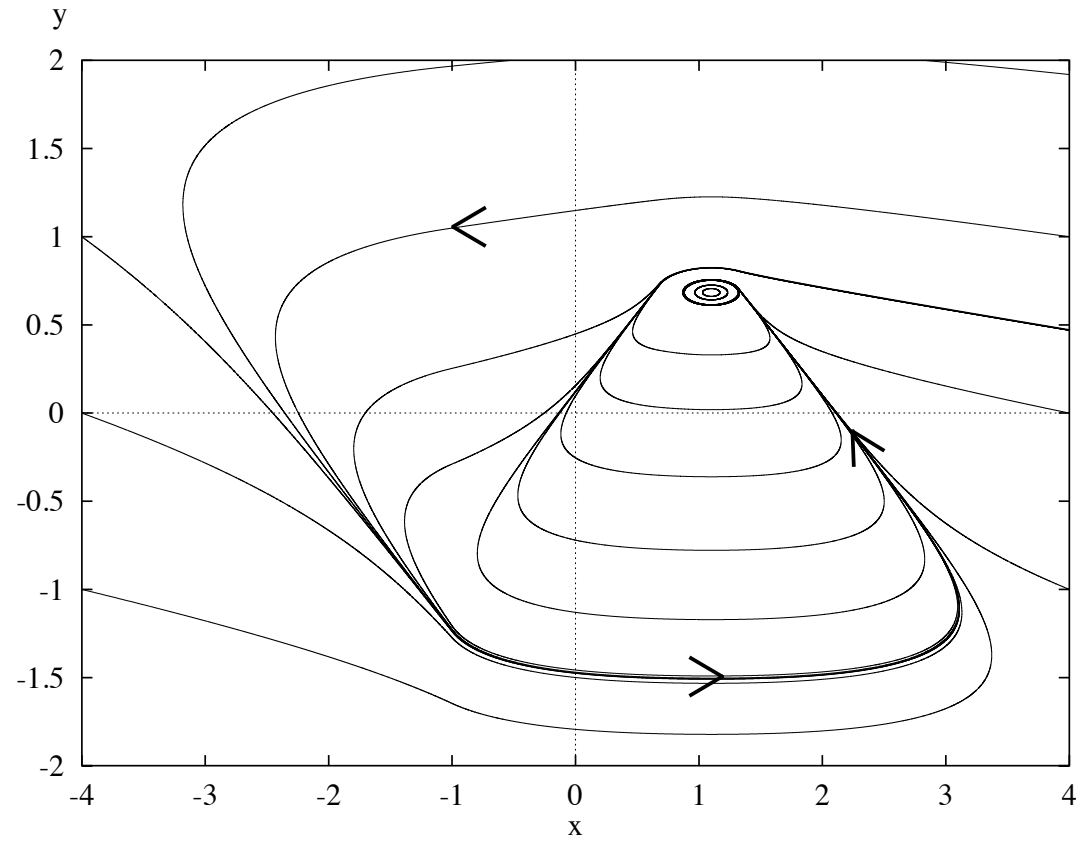

Figure 7: Phase plane of fast system (1) when the $y$-nullcline is fixed at $x=1.1$ and $\delta=0.1,(a \in(1,1+\sqrt{\delta}))$. The system has an attractive zonal linear center completely contained in the extra zone, and bounded by the periodic orbit tangent to the boundary $x=1+\sqrt{\delta}$. 


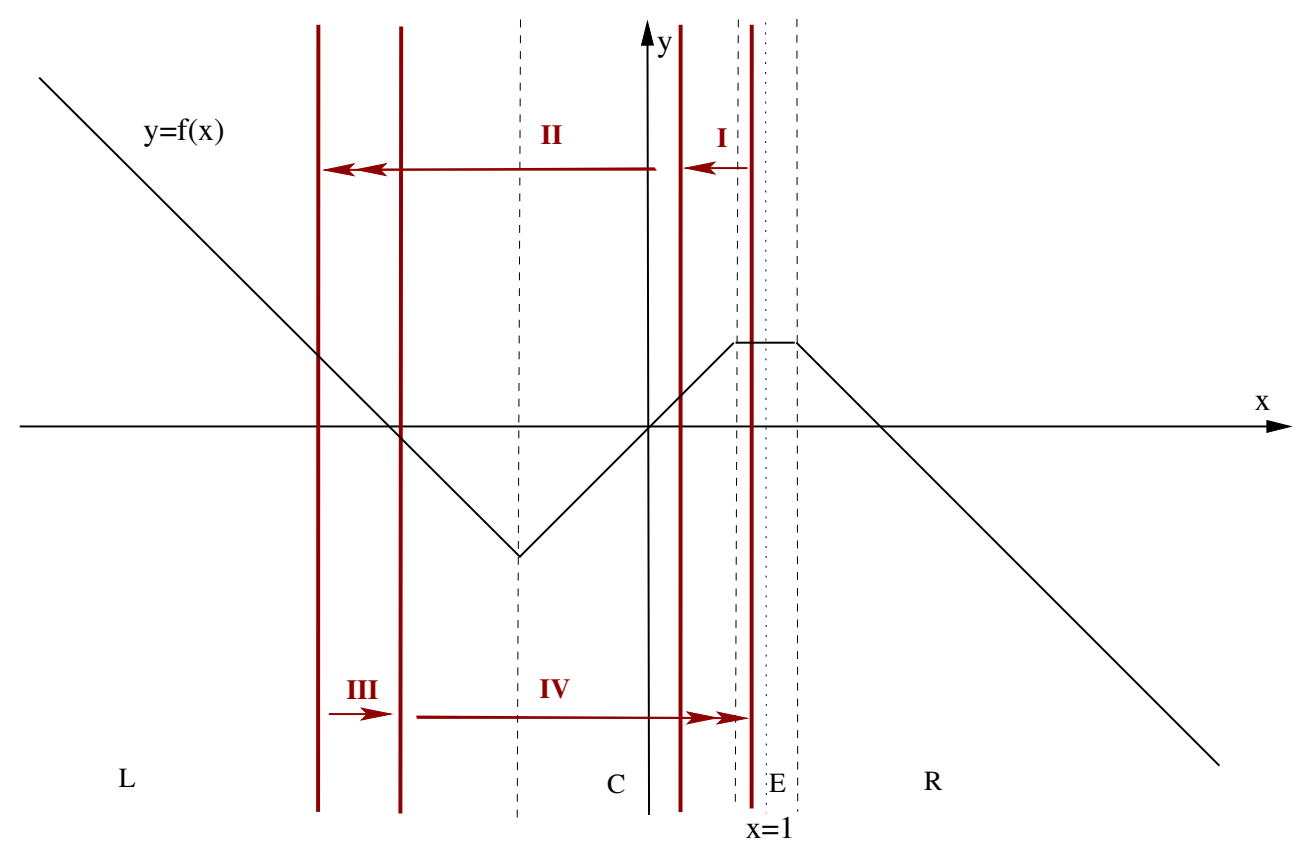

Figure 8: Sketch of the location of the $y$-nullcline when $c<c_{\min }$. In this case the limit cycle does not display small oscillations during the pause. 


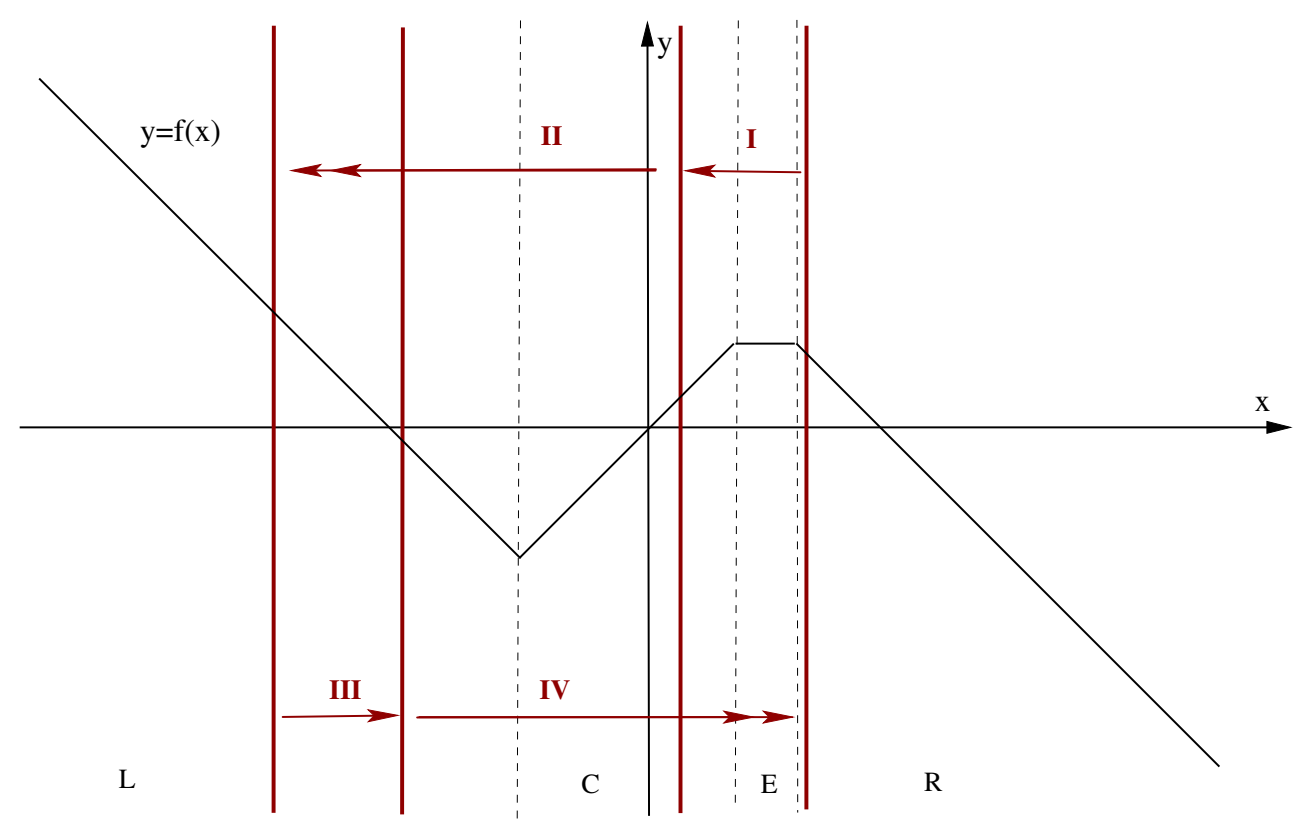

Figure 9: Sketch of the location of the $y$-nullcline when $c>c_{\max }$, giving rise to the maximal number of small oscillations. 


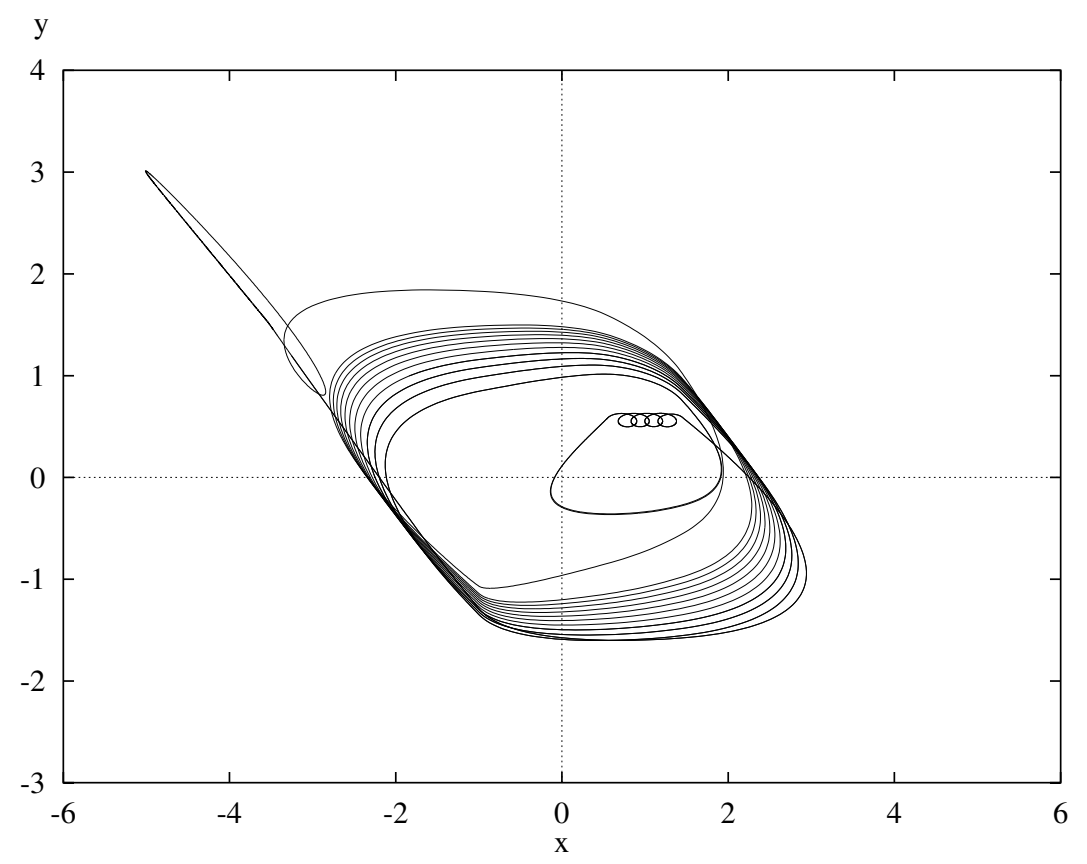

Figure 10: Phase plane in $x, y$ variables of the limit cycle of system (1)-(2) at the time of the passage through a secondary canard. Canard-shaped oscillations are produced between the pause and pulsatile regime. The parameter values are $a_{2}=2, b_{1}=0, b_{2}=0.5$, $k_{2}=0.6, \varepsilon=0.025, \delta=0.2$ and $c=1.4951$. 


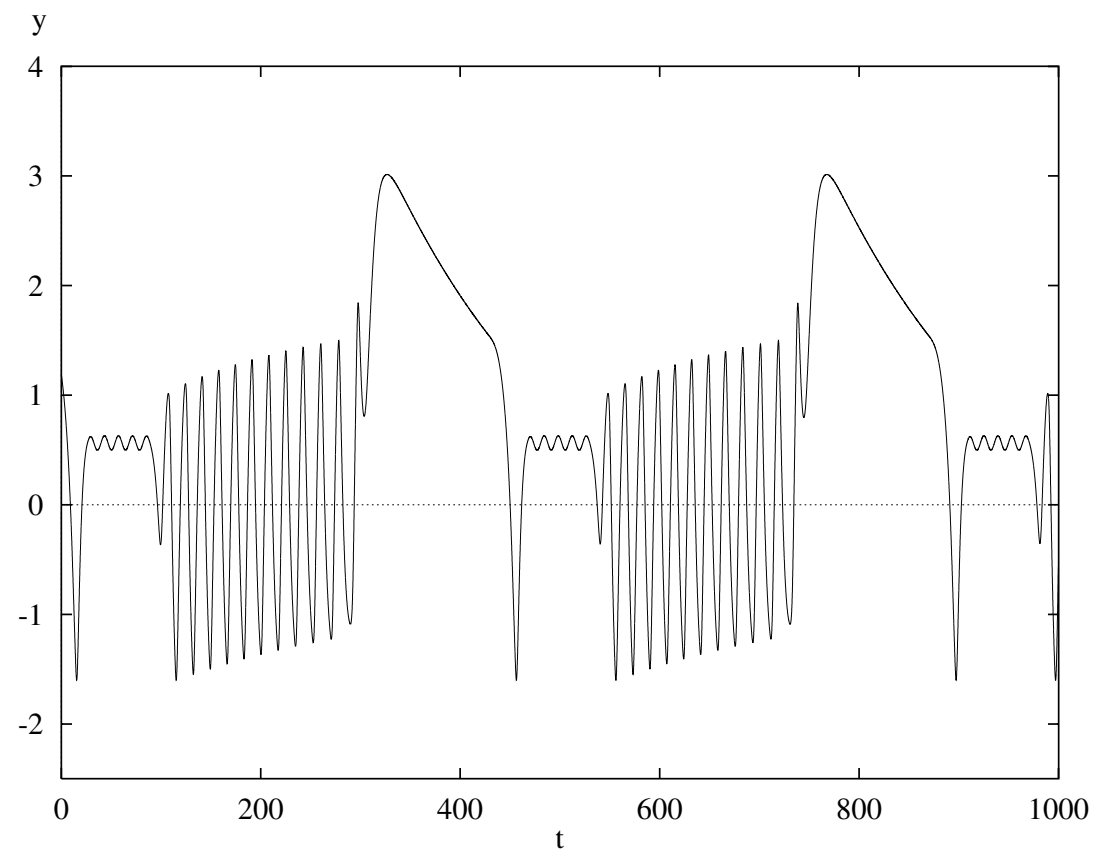

Figure 11: Second component of the limit cycle of system (1)-(2) at the time of passage through a secondary canard. An intermediate oscillation is being inserted between the pause and pulsatile regime. The parameter values are $a_{2}=2, b_{1}=0, b_{2}=0.5, k_{2}=0.6$, $\varepsilon=0.025, \delta=0.2$ and $c=1.4951$. 


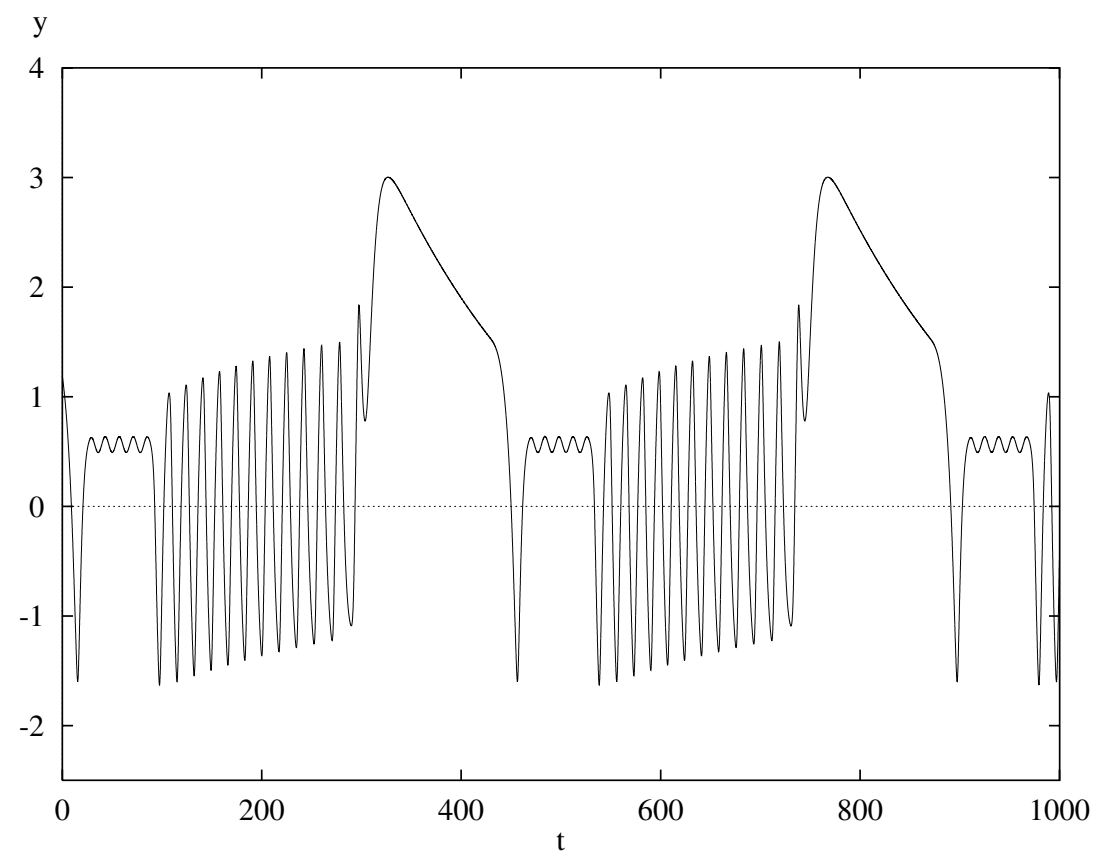

Figure 12: Second component of the limit cycle of system (1)-(2) after the passage through a secondary canard. The parameter values are $a_{2}=2, b_{1}=0, b_{2}=0.5, k_{2}=0.6, \varepsilon=0.025$, $\delta=0.2$ and $c=1.49$. Note that the limit cycle displays one small oscillation less than in Fig. 1. Compared to that figure, the only parameter that has been changed is $c$, which has decreased from 1.50 to 1.49 . 


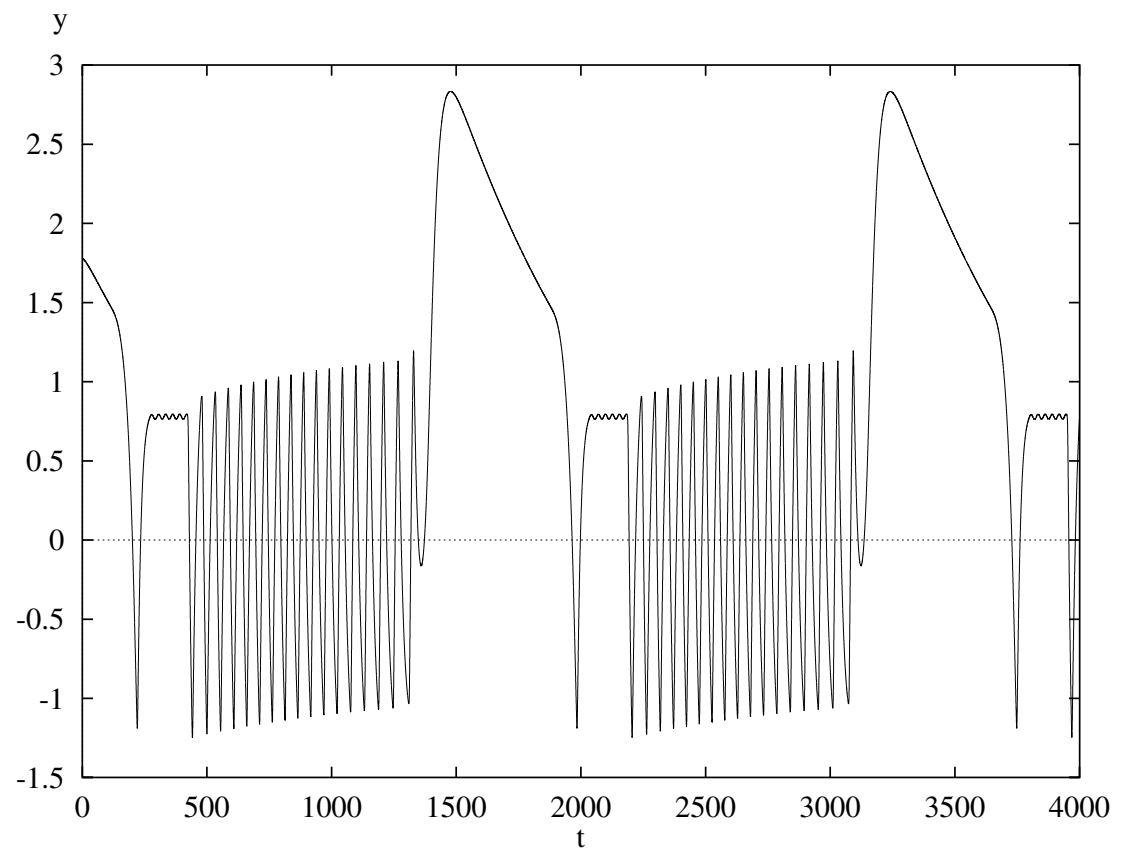

Figure 13: Second component of the limit cycle of system (1)-(2) for the parameter values $a_{2}=2, b_{1}=0, b_{2}=0.5, k_{2}=0.6, \varepsilon=0.025, \delta=0.05$ and $c=1.42$. The orbit displays 5 small oscillations during the pause and 17 pulses. 


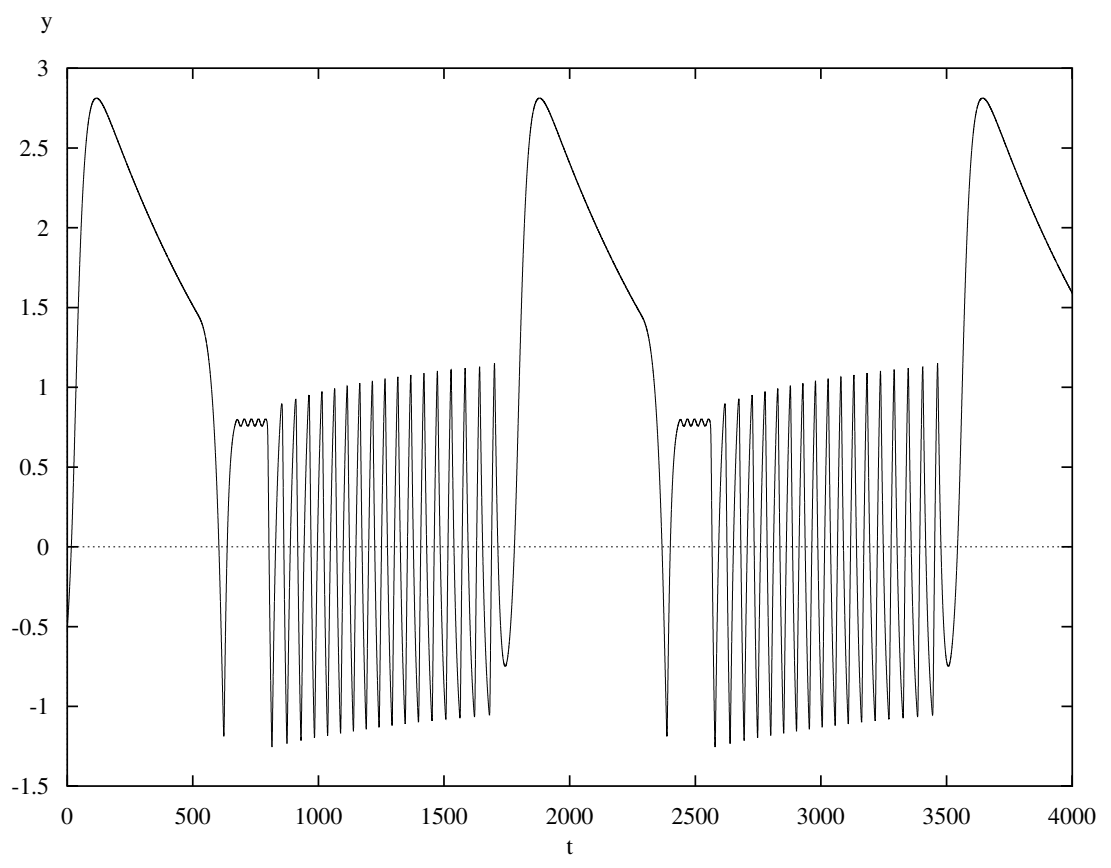

Figure 14: Second component of the limit cycle of system (1)-(2) for the parameter values $a_{2}=2, b_{1}=0, b_{2}=0.5, k_{2}=0.6, \varepsilon=0.025, \delta=0.05$ and $c=1.41$. The orbit displays 4 small oscillations and 17 pulses. Compared to the situation represented in Fig. 13, the limit cycle has passed through a secondary canard, and lost one small oscillation. However, in this case the loss is no more compensated for by the adding of one pulse. 


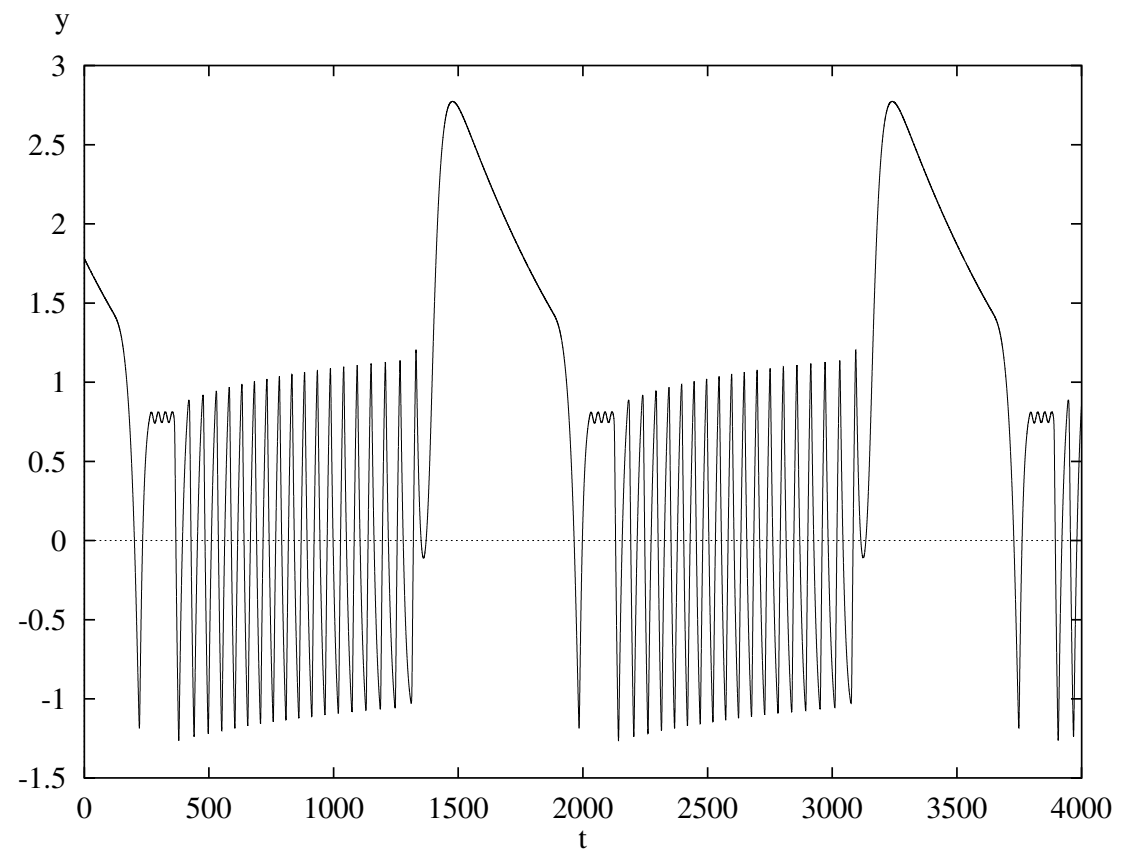

Figure 15: Second component of the limit cycle of system (1)-(2) for the parameter values $a_{2}=2, b_{1}=0, b_{2}=0.5, k_{2}=0.6, \varepsilon=0.025, \delta=0.05$ and $c=1.39$. The orbit displays 3 small oscillations and 18 pulses. Compared to the situation represented in Fig. 13, the limit cycle has passed through two secondary canards, and lost two small oscillations. The loss has been compensated for by the adding of one pulse. 